\title{
Structural-Functional Properties of Identified Excitatory and Inhibitory Interneurons within Pre-Bötzinger Complex Respiratory Microcircuits
}

\author{
Hidehiko Koizumi, Naohiro Koshiya, Justine X. Chia, Fang Cao, Joseph Nugent, Ruli Zhang, and Jeffrey C. Smith \\ Cellular and Systems Neurobiology Section, National Institute of Neurological Disorders and Stroke, National Institutes of Health, Bethesda, Maryland \\ 20892
}

We comparatively analyzed cellular and circuit properties of identified rhythmic excitatory and inhibitory interneurons within respiratory microcircuits of the neonatal rodent pre-Bötzinger complex (pre-BötC), the structure generating inspiratory rhythm in the brainstem. We combined high-resolution structural-functional imaging, molecular assays for neurotransmitter phenotype identification in conjunction with electrophysiological property phenotyping, and morphological reconstruction of interneurons in neonatal rat and mouse slices in vitro. This approach revealed previously undifferentiated structural-functional features that distinguish excitatory and inhibitory interneuronal populations. We identified distinct subpopulations of pre-BötC glutamatergic, glycinergic, GABAergic, and glycine-GABA coexpressing interneurons. Most commissural pre-BötC inspiratory interneurons were glutamatergic, with a substantial subset exhibiting intrinsic oscillatory bursting properties. Commissural excitatory interneurons projected with nearly planar trajectories to the contralateral pre-BötC, many also with axon collaterals to areas containing inspiratory hypoglossal (XII) premotoneurons and motoneurons. Inhibitory neurons as characterized in the present study did not exhibit intrinsic oscillatory bursting properties, but were electrophysiologically distinguished by more pronounced spike frequency adaptation properties. Axons of many inhibitory neurons projected ipsilaterally also to regions containing inspiratory XII premotoneurons and motoneurons, whereas a minority of inhibitory neurons had commissural axonal projections. Dendrites of both excitatory and inhibitory interneurons were arborized asymmetrically, primarily in the coronal plane. The dendritic fields of inhibitory neurons were more spatially compact than those of excitatory interneurons. Our results are consistent with the concepts of a compartmental circuit organization, a bilaterally coupled excitatory rhythmogenic kernel, and a role of pre-BötC inhibitory neurons in shaping inspiratory pattern as well as coordinating inspiratory and expiratory activity.

\section{Introduction}

Neural networks generating rhythmic movements contain core microcircuits of excitatory and inhibitory interneurons, the basic neural substrates for producing motor behavior (Grillner, 2006). Identifying intrinsic electrophysiological, morphological, and network properties of these interneurons is key to understanding the structural-functional organization of motor circuits. Here we have addressed this problem in a functionally defined, vital core circuit within brainstem networks generating breathing movements in mammals.

Rhythmic respiratory activity originates within excitatory and inhibitory circuits in the bilateral ventral respiratory column (VRC) of the medulla. Distinct VRC circuits are postulated to subserve specialized functions (Smith et al., 2007; Alheid and

\footnotetext{
Received Sept. 14, 2012; revised Dec. 14, 2012; accepted Dec. 17, 2012.

Author contributions: H.K. and J.C.S. designed research; H.K., N.K., J.X.C., and R.Z. performed research; H.K., N.K., J.X.C., F.C., J.N., R.Z., and J.C.S. analyzed data; H.K., N.K., J.X.C., and J.C.S. wrote the paper.

This research was supported by the Intramural Research Program of the NIH, National Institute of Neurological Disorders and Stroke. We thank Professor Yuchio Yanagawa of Gunma University, Japan for generously providing GAD67-GFP knock-in mice.

Correspondence should be addressed to Dr. Jeffrey C. Smith, 49 Convent Drive, Room 2A10, NINDS, NIH, Bethesda, MD 20892. E-mail: smithj2@helix.nih.gov.

DOI:10.1523/JNEUROSCI.4427-12.2013

Copyright $\odot 2013$ the authors $\quad 0270-6474 / 13 / 332994-16 \$ 15.00 / 0$
}

McCrimmon, 2008). Circuits within the pre-Bötzinger complex (pre-BötC), a functionally defined subregion of the VRC, generate and transmit the rhythmic activity producing inspiration (Smith et al., 1991; Rekling and Feldman, 1998). While there has been immense interest in rhythmogenic properties of pre-BötC microcircuits over the past two decades, structural-functional properties of the constituent neurons remain largely undefined. Subpopulations of rhythmic glutamatergic, glycinergic, and GABAergic pre-BötC neurons exist, and there is extensive indirect evidence that excitatory glutamatergic neuronal circuits are critical for rhythm generation (Koshiya and Smith, 1999; Tan et al., 2008; Bouvier et al., 2010; Gray et al., 2010). However, the biophysical/electrophysiological and structural properties of identified excitatory neurons have not been delineated. Furthermore, these properties of excitatory and inhibitory interneurons have not been differentiated. Such differentiation may distinguish their functions in rhythmogenesis and the basic operation of pre-BötC microcircuits.

Based on previous in vitro cellular- and circuit-level activity imaging, electrophysiological, and morphological analyses, we have postulated that a key subset of rhythmogenic excitatory neurons are commissural neurons, which interconnect and synchronize activity of pre-BötC interneurons on either side of the 
medulla (Koshiya and Smith, 1999; Koizumi et al., 2008). This concept has been supported more recently by genetic-based disruption of commissural connections in a set of transcription factor Dbx1-derived glutamatergic neurons (Bouvier et al., 2010), although this and our previous studies did not examine correlations between electrophysiological and structural properties. Such correlations have also not been pursued in recent studies of pre-BötC inhibitory neurons in slices from transgenic mice with fluorescent protein labeling of glycinergic or GABAergic neurons (Kuwana et al., 2006; Winter et al., 2009; Morgado-Valle et al., 2010). Here, we have used molecular identification of excitatory and inhibitory transmitter phenotypes, which in combination with electrophysiological and structural analyses allowed us to comparatively establish such features of interneurons studied in neonatal rodent in vitro slice preparations.

Our results identify structural-functional properties that distinguish excitatory and inhibitory subpopulations in the preBötC. Our findings are consistent with the concept of a compartmentalized function of the pre-BötC (Smith et al., 2007), and the concept that pre-BötC microcircuits incorporate a core set of bilaterally coupled excitatory interneurons for generating and transmitting rhythmic inspiratory drive (Butera et al., 1999b; Koshiya and Smith, 1999; Smith et al., 2000). Our results also suggest that inhibitory interneurons are organized to provide feedforward inhibition and potentially function to coordinate inspiratory and expiratory activity (Smith et al., 2009) in respiratory circuits.

\section{Materials and Methods}

Animal procedures. All animal procedures were approved by the NINDS Animal Care and Use Committee.

Medullary slice preparations. All electrophysiological and live neuron imaging experiments were performed with rhythmically active in vitro slice preparations (250-350 $\mu \mathrm{m}$ thick transverse slices) cut from the medulla oblongata from neonatal [postnatal day 1 (P1) to P3] Sprague Dawley rats of either sex or from P4 to P8 GAD67-GFP $(\Delta$ neo $)$ knock-in mice of either sex (Tamamaki et al., 2003) to contain the pre-Bötzinger complex and rostral end of the hypoglossal (XII) motor nucleus with intact XII nerve rootlets. The caudal surface of the slice was cut through the caudal pre-BötC for imaging and recording neuronal activity as previously described (Koizumi et al., 2008). The slice was oriented caudal end up in a recording chamber $(0.2 \mathrm{ml})$ mounted on the stage of an upright microscope and superfused $(4 \mathrm{ml} / \mathrm{min})$ with artificial CSF (ACSF) containing the following (in $\mathrm{mm}$ ): $124 \mathrm{NaCl}, 25 \mathrm{NaHCO}_{3}, 3 \mathrm{KCl}$, $1.5 \mathrm{CaCl}_{2}, 1.0 \mathrm{MgSO}_{4}, 0.5 \mathrm{NaH}_{2} \mathrm{PO}_{4}, 30$ D-glucose, and antibiotics (500 $\mathrm{U} / \mathrm{L}$ penicillin, $0.5 \mathrm{mg} / \mathrm{L}$ streptomycin, and $1 \mathrm{mg} / \mathrm{L}$ neomycin), equilibrated with $95 \% \mathrm{O}_{2}$ and $5 \% \mathrm{CO}_{2}\left(\mathrm{pH}=7.35-7.40\right.$ at $\left.27^{\circ} \mathrm{C}\right)$. During recording and imaging experiments rhythmic respiratory network activity in the XII nerves was maintained by elevating the superfusate $\mathrm{K}^{+}$ concentration to $8-9 \mathrm{~mm}$.

Calcium-sensitive dye labeling and imaging of rhythmically active preBötC neurons. For microscopic functional identification of respiratory neurons for patch-clamp recording, we loaded pre-BötC neurons with $\mathrm{Ca}^{2+}$-sensitive fluorescent dyes by two methods. In most experiments, the neurons were retrogradely labeled through their contralaterally projecting axons using membrane semipermeable acetoxymethyl (AM) dyes microinjected in the midline region of the slice (Fig. $1 A$ ) as previously described (Koshiya and Smith, 1999). We used Calcium Green-1 AM $(\mathrm{CaG})$ or Oregon Green BAPTA-1 AM (OGB) (Invitrogen). The slice was incubated overnight $(12 \mathrm{~h})$ for transmembrane diffusion of the dyes into axons followed by retrograde labeling of neuronal somas. The slices served for optical and electrophysiological recordings optimally throughout the second day. In all other experiments, we microinjected the dyes directly into the pre-BötC to label cells nonselectively regardless of their axonal projections. The microinjection pipette (tip size: $2-3 \mu \mathrm{m}$ ) was placed at a depth of $150 \mu \mathrm{m}$ and $\sim 100 \mu \mathrm{m}$ away from the center of
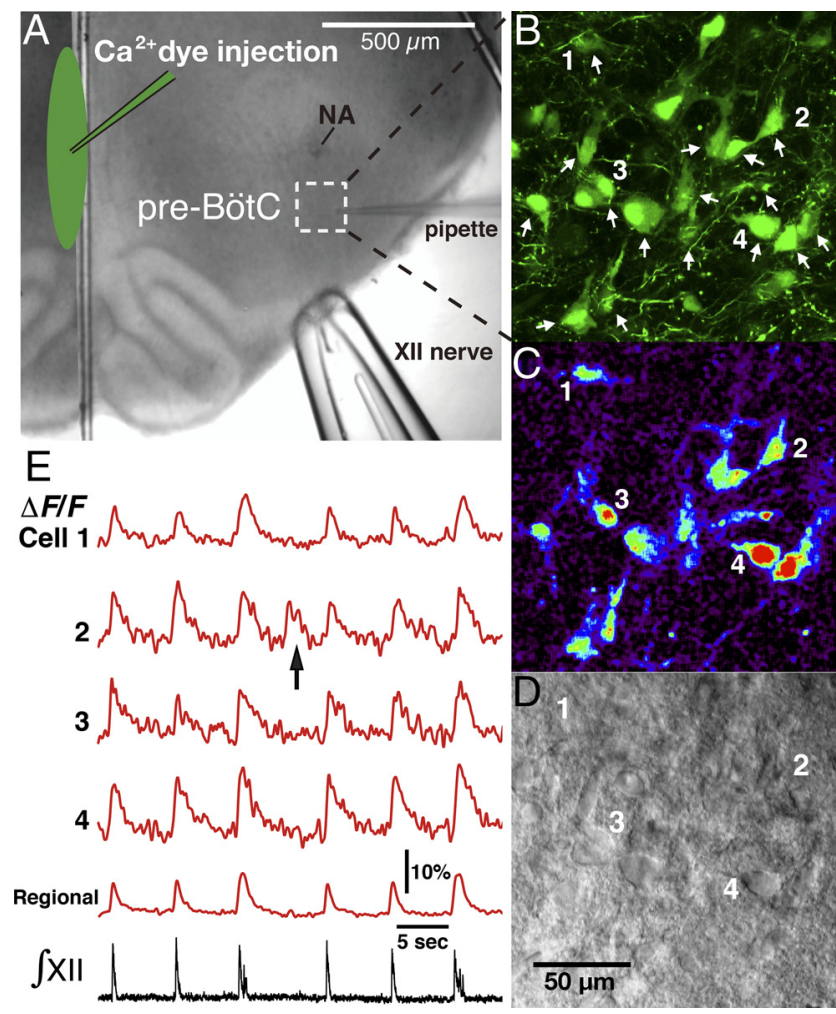

Figure 1. Overview of experimental in vitro neonatal rat rhythmic slice preparation with inspiratory neuronal activity imaging via $\mathrm{Ca}^{2+}$-sensitive fluorescent dye. $\mathrm{A}, \mathrm{Ca}^{2+}$. sensitive fluorescent dyes ( $\mathrm{Ca}^{2+}$ dyes) were injected along the midline of the slice (shown by infrared Dodt contrast structural image) to label pre-BötC neurons with contralaterally projecting axons. $\boldsymbol{B}$, Illustrative example of OGB labeled neurons in the pre-BötC area marked by dashed square in $\boldsymbol{A}$. Two-photon fluorescence, single optical section. Arrows indicate inspiratory neurons identified by analyzing their $\mathrm{Ca}^{2+}$ fluorescence signals in real time simultaneously with inspiratory hypoglossal nerve activity, which is used to monitor inspiratory network activity. Neurons labeled (1-4) are indicated in images and their fluorescence activity is shown in $\boldsymbol{C}$ and $\boldsymbol{E}$. C, Background-subtracted image from $\boldsymbol{B}$ showing inspiratory activity-related "flash" of increased fluorescence intensity during inspiration. $\boldsymbol{D}$, Dodt contrast image simultaneously captured with OGB fluorescent image in $\boldsymbol{B}$ and $\boldsymbol{C}$, providing structural view of individual neurons 1-4 and other neurons that exhibited inspiratory fluorescence transients. $\boldsymbol{E}$, Fluorescence intensity signals from cells 1-4 and from the captured pre-BötC region (spatially averaged fluorescence signal) showing $\mathrm{Ca}^{2+}$ transients synchronized with inspiratory hypoglossal nerve activities ( $\left.\int \mathrm{XII}\right)$. The arrow in cell 2 trace indicates an example of $\mathrm{Ca}^{2+}$ fluorescence transient associated with ectopic neuronal bursting independent of synchronized network activity as indicated by $\int \mathrm{XII}$ activity.

the pre-BötC to avoid excessive dye deposits and high background fluorescence in the imaging area of interest, and the dyes were pressure injected ( $\sim 20 \mathrm{psi}, 2 \mathrm{~min})$. The slice was incubated $(>1 \mathrm{~h})$ for sufficient dye loading before the experiments.

In an initial set of experiments, functional and structural imaging of labeled neurons was done with an intensifying fluorescent video microscopy system, the image from which was aligned with that of IR-DIC optics and obtained with a Newvicon camera as previously described (Koshiya and Smith, 1999; Koizumi et al., 2008). Most optical imaging experiments were done with a Leica multiphoton laser-scanning upright microscope (TCS SP5 II MP with DM6000 CFS system, LAS AF software, and a $20 \times$ water objective, N.A. 1.0). A Ti:sapphire pulsed laser (MaiTai, Spectra Physics) was used at $800-880 \mathrm{~nm}$ with DeepSee predispersion compensation. $\mathrm{Ca}^{2+}$-dye fluorescence imaging was done with the tandem resonant scanner ( $16 \mathrm{kHz}$ bidirectional, $\sim 25$ frames $/ \mathrm{s}$ for $512 \times 512$ pixel scan). Scan-range magnifications of $1.7-3 \times$ were typically used, and achieved by scan-range modifications, covering a $434 \times 434$ to $246 \times$ $246 \mu \mathrm{m}$ square, respectively. The two-photon fluorescence emission signal was detected by non-descanned detectors after a beam-splitter 
(560 nm, Leica original Semrock) and emission filter for the green calcium dyes (525/50, Semrock), and in some experiments for the red fluorescent dye (630/75, Chroma) (see section on morphological reconstruction from live imaging, below). Dynamic fluorescence images were acquired in real time along with electrophysiological signals of inspiratory hypoglossal nerve activities (LAS AF, Electrophysiology module ver. 2.60), and spatiotemporal features were visualized using IDL (Exelis). Simultaneous recording of these allowed us to functionally identify which pre-BötC inspiratory neurons were active in phase with inspiratory network activity. In all cases, average intensities of regions of interest were quantified for each frame, and dynamic fluorescence signals were normalized to baseline values and expressed as time courses of the ratio $\Delta F / F$. The infrared excitation laser for two-photon fluorescence was simultaneously used for transmission bright-field illumination to achieve a Dodt gradient contrast structural imaging to give fluorescence and structural images matched to pixels, allowing us to accurately place a patch pipette on functionally identified neurons. The conventional scanners of the Leica CFS system were also used for cellular morphological detection at a high spatial resolution up to $240 \mathrm{~nm}$ voxels. Three-dimensional (3D) two-photon optical stacks were acquired at $0.24-2.0 \mu \mathrm{m} \mathrm{Z}$ steps to determine the spatial distribution of labeled pre-BötC neurons at a depth up to $\sim 150 \mu \mathrm{m}$.

Electrophysiological recording. XII motoneuron population activity from XII nerves, used to monitor inspiratory network activity in the slice preparations, was recorded with firepolished glass suction electrodes $(60-120 \mu \mathrm{m}$ inner diameter), amplified (50,000-100,000 ×, CyberAmp 380, Molecular Devices), bandpass filtered $(0.3-2 \mathrm{kHz})$, digitized $(10 \mathrm{kHz})$ with an $\mathrm{AD}$ converter (Power Lab, ADInstruments), and then rectified and integrated either by an analog integrator or digitally with Chart software (ADInstruments).

Voltage- and current-clamp data were recorded with a HEKA EPC-9 or -10 patchclamp amplifier (HEKA Electronics) controlled by PatchMaster software (HEKA; $2.9 \mathrm{kHz}$ low-pass filter, sampled at $10 \mathrm{kHz}$ ). Recording electrodes (borosilicate glass pipette $4-6 \mathrm{M} \Omega$ ), positioned with microdrives [Scientifica or Siskiyou Design (SD) Instruments], contained (in $\mathrm{mm}$ ) $130.0 \mathrm{~K}$-gluconate, $5.0 \mathrm{Na}$-gluconate, 3.0 $\mathrm{NaCl}, 10.0 \mathrm{HEPES}, 4.0 \mathrm{Mg}$-ATP, $0.3 \mathrm{Na}-\mathrm{GTP}$, and 4.0 sodium phosphocreatine $(\mathrm{pH}=7.3$ using $\mathrm{KOH})$. In all cases measured potentials were corrected for the liquid junction potential $(-10 \mathrm{mV})$.

Analysis of intrinsic electrophysiological properties. Under current-clamp recording conditions, we tested functionally imaged and identified pre-BötC inspiratory neurons for intrinsic voltage-dependent oscillatory bursting properties (Butera et al., 1999a; Koshiya and Smith, 1999), and in neurons without these properties we characterized the time course of action potentials (AP) and repetitive spiking properties. To identify intrinsic oscillatory bursting neurons, we first applied steady depolarizing current with synaptic transmission intact and tested for ectopic bursting during the interval between synaptically driven inspiratory bursts (Fig. 2). If ectopic bursts were detected, we then blocked the excitatory synaptic transmission critical for respiratory network function in vitro by bath applying the non-NMDA glutamate receptor blocker, 6-cyano-7-nitroquinoxaline-2,3-dione disodium (CNQX) $\left(20 \mu \mathrm{M}\right.$; Sigma), and in some experiments by applying the $\mathrm{Ca}^{2+}$
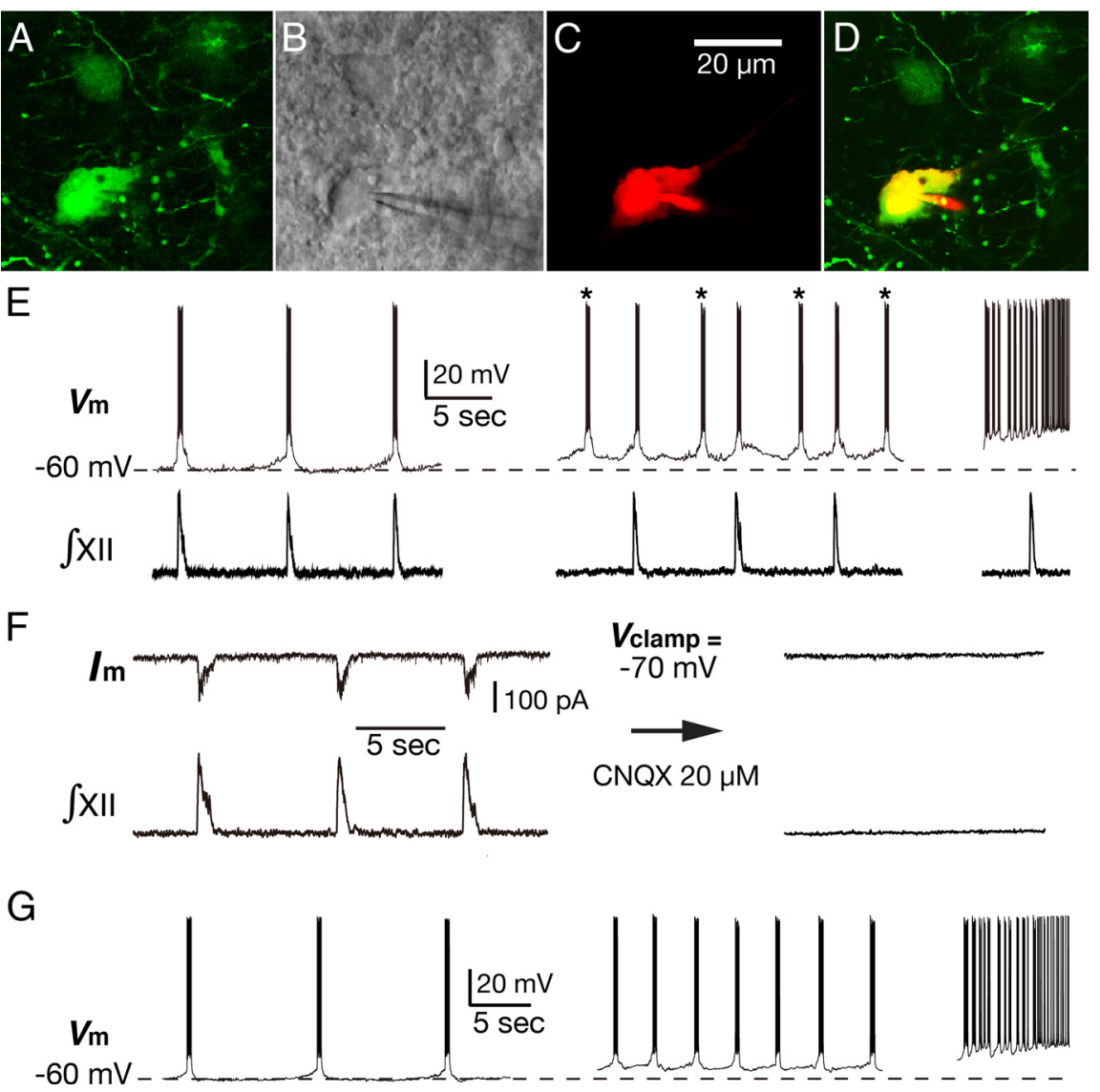

Figure 2. Illustration of voltage-dependent intrinsic oscillatory bursting behavior of pre-BötC inspiratory neuron under wholecell patch-clamp recording. $A$, Two-photon single optical plane image of the functionally identified pre-BötC inspiratory neurons exa 594 dye during whole-cell recordings. $\boldsymbol{D}$, Merged image of $\boldsymbol{A}$ and $\boldsymbol{C}$ confirming that the recorded neuron was the functionally inspiratory neuron from $\mathrm{Ca}^{2+}$ imaging. $\boldsymbol{E}$, Current-clamp recording from the imaged pre-BötC inspiratory $\mathrm{mV}$, neuronal bursting was synchronized with inspiratory network activity represented by $\int$ XII. Membrane depolarization to -55 dent of $\int$ XII rhythm. Further depolarization (right) induced higher frequency bursting and eventually tonic spiking. $\boldsymbol{F}$, Voltageclamp recording from the same neuron as in $\boldsymbol{E}$ showing rhythmic inward inspiratory synaptic drive currents, which were eliminated continuous rhythmic bursting after CNQX application and voltage-dependent intrinsic oscillatory bursting properties in which the bursting frequency is a function of the level of steady membrane depolarization.

channel blocker $\mathrm{Cd}^{2+}(200 \mu \mathrm{M}$; Sigma) to eliminate all synaptic transmission. Under these conditions we injected steady depolarizing current at several levels of membrane depolarization to confirm strong voltage-dependent intrinsic bursting behavior (Fig. 2). To analyze neuronal spiking properties we injected a depolarizing current pulse $(2 \mathrm{~ms} ; 0.5-1.2 \mathrm{nA})$ to evoke an AP and we specifically examined the amplitude and durations of medium afterhyperpolarizations (mAHP). The mAHP duration (average of 20 evoked APs) in each neuron analyzed was measured from a point where the membrane voltage was at baseline $V_{\mathrm{m}}$ immediately after the AP to the point at which the voltage reached this $V_{\mathrm{m}}$ again (Koizumi et al., 2008). We evoked repetitive spiking with a series of sustained current pulses ( $2-3 \mathrm{~s}$ duration) of increasing amplitude, and analyzed the instantaneous frequency of AP discharge, steady-state firing frequency, and spike frequency accommodation/adaptation. In both intrinsically bursting and intrinsically nonbursting neurons, we also tested for hyperpolarization-activated current $\left(I_{\mathrm{h}}\right)$ under current- and/or voltage-clamp conditions. For current-clamp recordings we applied from the baseline membrane potential a series of hyperpolarizing current injections (steps of $-0.05,-0.25$, or $-0.5 \mathrm{nA}$ depending on neuronal input resistance, $1 \mathrm{~s}$ step duration in all cases) to hyperpolarize the membrane potential in steps to $\sim-120 \mathrm{mV}$. For 
voltage-clamp recordings we applied $1 \mathrm{~s}$ hyperpolarizing voltage steps from $-65 \mathrm{mV}$ to $-120 \mathrm{mV}$.

We also applied voltage-clamp recording to analyze intrinsic membrane properties operating at subthreshold potentials in both types of pre-BötC inspiratory neurons under conditions where excitatory synaptic currents were blocked. We measured subthreshold voltage activating persistent sodium current $\left(I_{\mathrm{NaP}}\right)$, and we quantified leak currents $\left(I_{\text {Leak }}\right)$ by applying a slow depolarizing voltage-ramp protocol $(30 \mathrm{mV} / \mathrm{s} ;-110$ to $+10 \mathrm{mV})$ to generate current-voltage $(I-V)$ relations (Koizumi and Smith, 2008). In some cases, the protocols were repeated under bathapplied $1 \mu \mathrm{M}$ Tetrodotoxin (TTX, Sigma) to better isolate TTX-sensitive $\mathrm{Na}^{+}$currents by subtracting $I-V$ curves obtained before and after TTX application. Linear regression analysis applied to the essentially linear region of the quasi-steady-state voltage-clamp ramp $I-V$ relations was used to determine the neuronal membrane leak conductance $\left(g_{\text {Leak }}\right)$ as previously described (Koizumi and Smith, 2008). In some of these neurons, we also quantified the magnitude of $I_{\mathrm{h}}$ current from a series of hyperpolarizing voltage steps ( $1 \mathrm{~s}$ duration) from $-65 \mathrm{mV}$ to $-120 \mathrm{mV}$. In all neurons cell capacitance $\left(C_{\mathrm{m}}\right)$ was determined from the integral of the transient capacitance current $\left(I_{\mathrm{C}}\right.$, leak subtracted) evoked by a $15 \mathrm{~ms}$ hyperpolarizing voltage-step command $\left(\mathrm{d} V_{\mathrm{C}}\right)$ applied within $\sim 10 \mathrm{mV}$ of resting potential, using the formula $C_{\mathrm{m}}=\int I_{\mathrm{C}} / \mathrm{d} V_{\mathrm{C}}$. In some experiments, the $C_{\mathrm{m}}$ was obtained as the value used for capacitance compensation in voltage-clamp mode. Series resistance $\left(R_{\mathrm{S}}\right)$ was compensated online by $\geq 80 \%$, and the compensation was periodically readjusted. Neurons exhibiting clear evidence of poor space clamp such as unclamped AP currents during voltage-clamp measurements were excluded from the analysis.

Measurements of all parameters of cellular activity obtained from current-clamp or membrane properties derived from voltage-clamp were made off-line with custom automated algorithms [PowerLab with Chart software (ADInstruments) and Igor Pro (Wavemetrics)]. Statistical significance was determined with a two-tailed Student's $t$ test on mean data, and data are presented as means \pm SD.

Single-cell multiplex RT-PCR. After electrophysiological properties of the imaged pre-BötC inspiratory neurons were defined by whole-cell patch-clamp recording, the cytoplasm was aspirated as completely as possible into the patch pipette under visual control and then immediately expelled into a thin-walled tube for PCR-containing reverse transcription reagents (Invitrogen) (Koizumi et al., 2010). To avoid contamination, we used autoclaved capillary glasses for the electrodes, and also when the electrode was in the slice, we continuously applied positive pressure to the electrode, producing a continuous stream of pipette solution, to keep the pipette tip clean until establishing whole-cell recording and the cytoplasm harvesting was performed. Single-cell multiplex RT-PCR (scmRT-PCR) was subsequently performed on the cytoplasm to probe for mRNA expression of vesicular glutamate transporter 2 (VgluT2), glycine transporter 2 (GlyT2), and glutamic acid decarboxylase 67 (GAD67) to identify glutamatergic, glycinergic, or GABAergic neurons. First strand cDNA was synthesized for $1.5 \mathrm{~h}$ at $50^{\circ} \mathrm{C}$ in a mixture of $\mathrm{MgCl}_{2}(2 \mu \mathrm{l}, 25 \mathrm{~mm})$, dNTPs $(1 \mu \mathrm{l}, 10 \mathrm{~mm})$, BSA $(0.7 \mu \mathrm{l}, 143 \mathrm{ng} / \mu \mathrm{l})$, random hexamers $(1 \mu \mathrm{l}, 50 \mathrm{ng} / \mu \mathrm{l})$, oligodT $(0.7 \mu \mathrm{l}, 0.5 \mu \mathrm{g} / \mu \mathrm{l})$, RNaseOUT $(1.2 \mu \mathrm{l}, 40 \mathrm{u} / \mu \mathrm{l})$, DTT $(1 \mu \mathrm{l}, 0.1 \mathrm{M})$, and SuperScriptIII RT $(1 \mu \mathrm{l}$, $200 \mathrm{u} / \mu \mathrm{l})$. The entire reaction was either immediately used as template for multiplex PCR or frozen at $-80^{\circ} \mathrm{C}$ until assayed. Following reverse transcription, the cDNA for each target mRNA was amplified simultaneously in a multiplex PCR procedure, using primers for VgluT2 (forward, 5'-TGTTCTGGCTTCTGGTGTCTTACGAGAG-3'; reverse, 5' -TTCC CGACAGCGTGCCAACA-3'), GlyT2 (forward, 5' -TCTGCATGACTG CCTATCCG-3'; reverse, 5'-AACACAGGCTTGTGTGTGCG-3'), and GAD67 (forward, 5' -ACCCTGGTGCCCGCTTCC-3'; reverse, 5' -TATT GGTATTGGCAGTTGATGTC- $3^{\prime}$ ). The first multiplex PCR was performed as a hot start reaction in a final volume of $50 \mu \mathrm{l}$ containing $12 \mu \mathrm{l}$ reverse transcription reaction, $20-50$ pmol of each primer, $0.2 \mathrm{~mm}$ dNTPs, $10 \times$ High Fidelity PCR buffer with $2 \mathrm{mM} \mathrm{MgCl}_{2}$, and $5 \mathrm{U}$ of Platinum Taq High Fidelity DNA polymerase (Invitrogen). The reaction mixtures were heated to $94^{\circ} \mathrm{C}$ for $2 \mathrm{~min} ; 30$ cycles $\left(94^{\circ} \mathrm{C}, 30 \mathrm{~s} ; 55^{\circ} \mathrm{C}, 30 \mathrm{~s}\right.$; $68^{\circ} \mathrm{C}, 1 \mathrm{~min}$ ) of PCR were followed by a final elongation period of $10 \mathrm{~min}$ at $68^{\circ} \mathrm{C}$. The second round of PCR amplification was performed as indi- vidual reactions with individual primers for VgluT2 (forward, 5'-AGGT ACATAGAAGAGAGCATCGGGGAGA-3'; reverse, 5' -CAC TGTAGTT GTTGAAAGAATTTGCTTGCTC-3'), GlyT2 (forward, 5' -TCTGCATG ACTGCCTATCCG-3'; reverse, 5' -CATGGTGTCAAGTCCAAGCG$3^{\prime}$ ), and GAD67 (forward, 5'-GGACTTCCACCACCCACAC-3'; reverse, $5^{\prime}$-CTAAACCAATGATATCCAAACCAG-3'), using $1 \mu \mathrm{l}$ of the first PCR product under similar conditions with the following modifications: 50 pM of each primer pair and 25 thermal cycles. Aliquots $(10 \mu \mathrm{l})$ of PCR products were separated and visualized in a SYBR Green-stained agarose gel (2\%) by electrophoresis. The expected sizes of PCR-generated fragments were as follows: VgluT2 (315 bp), GlyT2 (701 bp), and GAD67 (185 bp). To ensure that the PCR signal arose from the cytoplasm of the recorded cell, the same RT-PCR assays were run on pipette solution collected from negative control "mock harvests" in each experiment. These assays were performed by introducing a pipette into the slice without extracting cell contents. In all scmRT-PCR assays, $100 \mathrm{pg}$ of total rat brain RNA (Ambion) was also run as RT template to serve as a positive control.

Immunohistochemical labeling. To complement the molecular analysis by scmRT-PCR, we performed fluorescence immunolabeling directly for glycine or GABA in a separate set of neurons that were labeled with $\mathrm{Ca}^{2+}$-sensitive dye, imaged, and electrophysiologically identified. These pre-BötC inspiratory neurons were filled with biocytin $(0.5-0.8 \%)$ in the patch-pipette solution during whole-cell electrophysiological recordings. The slices were subsequently fixed in $4 \%$ paraformaldehyde (w/v) in phosphate-saline buffer, and cryoprotected overnight at $4^{\circ} \mathrm{C}$ in $30 \%$ sucrose, $0.1 \mathrm{~m}$ PBS solution and resectioned coronally at $50 \mu \mathrm{m}$ on a freezing microtome. In some sets of experiments, we made whole-mount preparations of the slices to immunolabel superficial neurons studied electrophysiologically. For fluorescent immunohistochemistry, floating sections or whole slices were incubated with $10 \%$ donkey serum in PBS with Triton X-100 (0.3\%) and subsequently incubated for $48-72 \mathrm{~h}$ at room temperature with the following primary antibodies: rabbit antiGlycine (1:2000; Immunosolution), or rabbit anti-GABA (1:1000; Sigma) and goat anti-choline acetyltransferase (ChAT) (1:500; Millipore Bioscience Research Reagents). Individual sections or whole slices were then rinsed with PBS and incubated for $2 \mathrm{~h}$ with the secondary antibodies Donkey anti-Rabbit IgG conjugated with Dylight 649 (1:500; Jackson ImmunoResearch) or Donkey anti-Goat IgG conjugated with Rhodamine Red-X (1:500; Jackson ImmunoResearch). Biocytin-filled cells were revealed using Avidin conjugate with Texas Red or Alexa Fluor 488 (1:500; Invitrogen). Individual sections were mounted on slides and covered with an anti-fading medium (Fluoro-Gel; Electron Microscopy Sciences). Whole-mounts (Koizumi et al., 2008) were placed in well slides with the slice surface containing labeled neurons appropriately oriented for imaging and then coverslipped. Fluorescent labeling of biocytin-filled neurons was visualized with a laser-scanning confocal imaging system (Zeiss LSM 510). Glycinergic and GABAergic cell bodies were identified by the presence of immunoreactivity for glycine or GABA antibody without ChAT antibody labeling (to confirm that the labeled neurons were not motoneurons). As a negative control for immunoreactivity, we obtained fluorescent images of biocytin-filled neurons where the slices were treated with secondary but not primary antibodies. All images were color/contrast enhanced and adjusted with a thresholding filter in Adobe Photoshop.

Morphological reconstruction of pre-BötC inspiratory neurons from slice whole-mounts. We histologically reconstructed and analyzed neuron location, somato-dendritic morphological features, and axonal projection patterns in slices containing biocytin-filled $(0.5-$ $1.5 \%)$ pre-BötC inspiratory neurons that were processed in wholemounts of the slice with a DAB-based reaction. All of these neurons were first labeled and imaged with fluorescent $\mathrm{Ca}^{2+}$-sensitive dye and analyzed electrophysiologically. For each electrophysiologically recorded neuron, we simultaneously captured the Dodt-contrast structural image and the cell fluorescence image, obtained by filling the cell with Alexa 594 hydrozide dye $\left(50 \mu \mathrm{M}, \mathrm{Na}^{+}\right.$salt, Invitrogen $)$also included in the patch solution, allowing us to establish the precise location and identify each recorded neuron in the subsequent histological analysis. To ensure neurotracer diffusion into longer dendrites 
and axons, we incubated the slice for at least $2 \mathrm{~h}$ after filling with biocytin. For biocytin visualization, the slices were fixed and rinsed as described above, and then pretreated for 30 min with $0.3 \% \mathrm{H}_{2} \mathrm{O}_{2}$ in PBS with $0.5 \%$ Triton X-100. Whole slices were then incubated overnight at $4^{\circ} \mathrm{C}$ in an avidin-biotinylated horseradish peroxidase reagent (Vectastain, Vector Laboratories), rinsed, and developed with nickelintensified DAB reaction (DAB kit, Vector Laboratories). The slices were dehydrated, cleared with methylsalicylate, mounted in well slides, coverslipped, and coated with Permount (Fisher Scientific). We then acquired high-resolution images of biocytin-filled neurons with a CCD camera $(1392 \times 1040$ resolution, typically 0.93 or 1.87 $\mu \mathrm{m}$ pixels with the $20 \times$ (or a $10 \times$ ) objective and a $0.35 \times$ reduction lens with the microscope (Leica TCS SP5 II) scanning at multiple focal planes (thickness $\leq 1 \mu \mathrm{m}$ ) in relevant regions of the slice, and reconstructed somato-dendritic morphology and axonal projections throughout the entire slice with Neurolucida software (ver. 10.42/10.5.02, MBF Bioscience). We then digitally merged the images with Neurolucida Montage module into a master $3 \mathrm{D}$ image with $2 \mathrm{D}$ projections to reconstruct neuronal location, somatodendritic morphology, and axonal projection pattern.

Morphological reconstructions from live imaging of neurons in situ. In a separate set of experiments, we obtained morphological information from live images of pre-BötC inspiratory neurons in situ in the in vitro slice preparations. We fluorescently visualized neuron morphology during whole-cell recordings by adding Alexa 594 hydrozide dye $(50 \mu \mathrm{M}$, $\mathrm{Na}^{+}$salt, Invitrogen) into the patch solution, and after adequate dye diffusion to visualize dendritic and axonal processes, we obtained stacks of two-photon images containing multiple optical sections ( 0.25 to $\sim 2.00 \mu \mathrm{m}$ thickness) with the Leica LAS software. Morphological reconstructions of the Alexa dye-labeled neurons were subsequently produced in Neurolucida interactively with the Autoneuron 3D reconstruction module.

Morphometric analyses. All quantitative morphometric analyses of individual neurons presented were conducted with Neurolucida Explorer ver.10.42 (MBF bioscience) and L-Measure software ver. 4.0 (Scorcioni et al., 2008). Statistical significance was determined with an unpaired Student's $t$ test on mean data, and data are presented as means $\pm S D$. These reconstructions may not represent the full $3 \mathrm{D}$ features of dendritic fields as they exist in the intact brainstem because some dendrites are likely cut at the surface of the in vitro slice preparations in which we obtained the electrophysiological measurements/ molecular assay for correlation with morphological properties. Neurons with obvious truncation of dendrites were excluded from the analyses. Our primary objective for the morphological reconstructions was to identify unequivocal and major preserved features of dendritic morphology of excitatory and inhibitory pre-BötC inspiratory neurons.

\section{Results \\ $\mathrm{Ca}^{2+}$-sensitive dye-labeled pre-BötC inspiratory neurons consisted of intrinsic bursters and intrinsically nonburster neurons}

Pre-BötC inspiratory neurons retrogradely labeled by midline injection of $\mathrm{Ca}^{2+}$-sensitive dyes

We imaged pre-BötC neurons labeled with $\mathrm{Ca}^{2+}$-sensitive dyes from midline injection at depths of up to $150 \mu \mathrm{m}$ in the slices, and identified the rhythmically active inspiratory neurons that exhibited rhythmic $\mathrm{Ca}^{2+}$ fluorescence transients in phase with the rhythmic motoneuronal population activity recorded from XII nerve roots, which was used to monitor inspiratory network activity in the slices (Fig. 1). These rhythmic neurons represented $60-70 \%$ of the total population of retrogradely labeled neurons. Under current-clamp recordings, all optically identified preBötC inspiratory neurons that were subsequently phenotyped (below) for neurotransmitter expression by scmRT-PCR or separately by immunostaining ( $n=232$ total from 64 slices) exhibited spike discharges synchronized with the rhythmic XII nerve inspiratory activities (Fig. 2). These pre-BötC inspiratory neurons had two distinct electrophysiological profiles, as previously reported (Koshiya and Smith, 1999; Del Negro et al., 2002b): neurons exhibited either intrinsic voltage-dependent oscillatory bursting (referred to hereafter as intrinsic bursters, $n=81,35 \%$ of total) (Fig. 2) or only tonic spiking (referred to as nonbursters, $n=151,65 \%$ ), when the baseline potential was depolarized by applying steady depolarizing bias current. The intrinsic bursters were characterized by prominent ectopic bursting during the silent period between XII inspiratory bursts following depolarization under current-clamp recording (Fig. 2). To confirm robust voltage-dependent intrinsic bursting behavior, in a subset of these neurons we blocked excitatory synaptic transmission by bath application of the non-NMDA glutamate receptor antagonist CNQX (20 $\mu \mathrm{M}, n=34)$ (Koshiya and Smith, 1999), which completely eliminated the neuronal inspiratory-related rhythmic synaptic drive currents (Fig. 2) and rhythmic inspiratory XII motor activity in the slice. Under these conditions, all neurons identified as intrinsic bursters exhibited oscillatory bursting behavior as illustrated in Figure 2G. In some cases we blocked all synaptic transmission with the voltage-dependent $\mathrm{Ca}^{2+}$ channel blocker $\mathrm{Cd}^{2+}(200 \mu \mathrm{M}, n=6)$, and confirmed that the neurons tested exhibited the same type of intrinsic bursting behavior observed after block of excitatory synaptic transmission alone.

\section{Pre-BötC inspiratory neurons labeled by local injection of} $\mathrm{Ca}^{2+}$-sensitive dyes

We also used local injection of $\mathrm{Ca}^{2+}$-sensitive dyes to label preBötC neurons to obtain a sample of inspiratory neurons independent of axonal projection patterns, specifically to include noncommissural neurons that may also be inhibitory neurons, since our results (below) showed that the majority of neurons with midline-crossing axons were excitatory neurons. Local injection of $\mathrm{CaG} / \mathrm{OGB}$ into the pre-BötC was effective for labeling inspiratory neurons exhibiting rhythmic $\mathrm{Ca}^{2+}$ fluorescence transients at depths up to $\sim 150 \mu \mathrm{m}$. While all of these optically identified pre-BötC inspiratory neurons ( $n=39$ total in 8 slices) exhibited spike discharges synchronized with XII inspiratory activities under current-clamp recordings, only $15 \%(n=6 / 39)$ of the pre-BötC neurons sampled by this approach exhibited intrinsic oscillatory bursting properties. Previous estimates of the fractional composition of intrinsic bursters as identified within the pre-BötC inspiratory neuron population by different sampling approaches (not based on axonal projections) range from $\sim 5 \%$ (Del Negro et al., 2002a) to 25\% (Peña et al., 2004; Pagliardini et al., 2005) of the total inspiratory population. Thus our estimates from sampling after neuronal labeling via local injection of $\mathrm{Ca}^{2+}$-sensitive dye are congruent with these other estimates. In contrast, our results from sampling based on midline-crossing axonal projections indicate that this population of inspiratory commissural neurons is relatively enriched with intrinsic burster neurons (35\% of total), which is also consistent with the results presented below for neurons with identified neurotransmitter phenotypes.

\section{Identification of glutamatergic, glycinergic, and GABAergic pre-BötC inspiratory neurons by single-cell multiplex RT-PCR}

In neurons retrogradely labeled with $\mathrm{Ca}^{2+}$-sensitive dye and functionally identified as inspiratory cells, we probed for mRNA expression of VgluT2, GlyT2, and GAD67 in the cytoplasm harvested from the neurons during whole-cell recording (Fig. 3) to identify glutamatergic, glycinergic, and GABAergic neurons, re- 


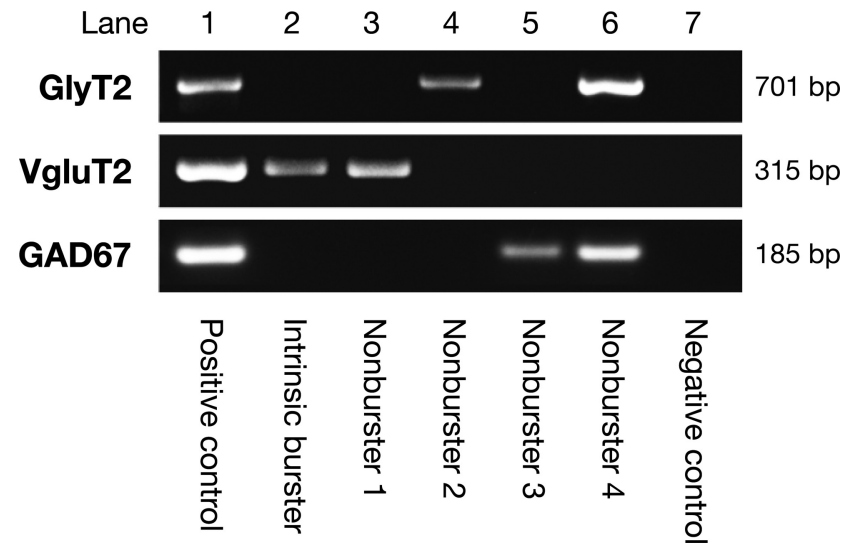

Figure 3. Identification of neurotransmitter phenotype of pre-BötC inspiratory neurons by single-cell multiplex RT-PCR. Electrophoresis gel generated by multiplex RT-PCR from mRNA in cytoplasm aspirated from 5 representative pre-BötC inspiratory neurons showing CDNA for VgluT2 expression by intrinsic burster neuron (lane 2), VgluT2 by intrinsic nonburster neuron (lane 3), GlyT2 (lane 4), GAD67 (lane 5), and both GlyT2 and GAD67 (lane 6), respectively, expressed by intrinsic nonburster neurons. The positive control (lane 1) from $100 \mathrm{pg}$ of total rat brain RNA (tRNA) shows the predicted base pair (bp) molecular weights of amplimers of VgluT2 (315 bp), GlyT2 (701 bp), and GAD67 (185 bp). No VgluT2, GlyT2, or GAD67 signals were present in negative controls (lane 7) from the "mock harvests."

Table 1. Summary of neurotransmitter-related mRNA expression of pre-BötC inspiratory neurons

\begin{tabular}{|c|c|c|c|c|c|c|}
\hline & Neuron type & VgluT2 & GlyT2 & GAD67 & GlyT2-GAD6 & Total \\
\hline \multirow{3}{*}{$\begin{array}{l}\text { Labeled by midline } \\
\text { injection }\end{array}$} & Intrinsic burster & $42(37 \%)$ & $0(0 \%)$ & $0(0 \%)$ & $0(0 \%)$ & $42(37 \%)$ \\
\hline & Nonburster & $49(43 \%)$ & $6(5 \%)$ & $5(4 \%)$ & 12 (11\%) & 72 (63\%) \\
\hline & Both typ & $91(80 \%)$ & $6(5 \%)$ & $5(4 \%)$ & 12 (11\%) & 114 (100\% \\
\hline \multirow{3}{*}{$\begin{array}{l}\text { Labeled by local } \\
\text { injection }\end{array}$} & Intrinsic burster & $6(15 \%)$ & $0(0 \%)$ & $0(0 \%)$ & $0(0 \%)$ & $6(15 \%)$ \\
\hline & Nonburster & $14(36 \%)$ & $4(10 \%)$ & $5(13 \%)$ & $10(26 \%)$ & $33(85 \%)$ \\
\hline & Both types & 20 (51\%) & $4(10 \%)$ & $5(13 \%)$ & $10(26 \%)$ & $39(100 \%$ \\
\hline
\end{tabular}

spectively. Only neurons with clean negative controls from "mock harvests" in the slice and appropriate positive controls (Fig. 3) were used for the analysis. For these neurons, no VgluT2, GlyT2, or GAD67 mRNA signals were detected in negative controls (Fig. 3). The sample population consisted of 114 neurons total, with 42 intrinsic bursters (37\%) and 72 nonburster inspiratory neurons $(63 \%)$. The results are summarized in Table 1. All intrinsic bursters expressed only VgluT2 mRNA $(n=42 / 42)$, while nonbursters $(n=72)$ exhibited mixed expression patterns. Subsets of the nonbursters expressed only VgluT2 mRNA ( $n=$ $49 / 72$, 68\%), only GlyT2 mRNA $(n=6 / 72,8 \%)$, only GAD67 mRNA $(n=5 / 72,7 \%)$, or both GlyT2 and GAD67 mRNA $(n=$ 12/72, 17\%); no VgluT2 mRNA expression was found in these different types of inhibitory neurons. Thus, of the nonbursters identified by retrograde labeling, 32\% $(n=23 / 72)$ were inhibitory neurons. Coexpression of GlyT2 and GAD67 mRNA in a single neuron is not uncommon (see Discussion). In summary, the majority ( $n=91 / 114,81 \%$ ) of pre-BötC inspiratory neurons with contralaterally projecting axons as identified by retrograde labeling were excitatory/glutamatergic neurons. Additionally, all intrinsic bursters identified were glutamatergic neurons. Intrinsic voltage-dependent bursting properties were not observed in any of the different types of inhibitory neurons.

We also assayed for mRNA expression in the population of pre-BötC inspiratory neurons $(n=39)$ labeled by locally injected $\mathrm{Ca}^{2+}$-sensitive dyes. In this sample, we identified 6 intrinsic bursters and 33 nonburster neurons (Table 1). All of these intrinsic bursters were excitatory, expressing only VgluT2 mRNA ( $n=$
6/6, 100\%). Again, nonbursters had four different expression patterns: VgluT2 mRNA only $(n=14 / 33,42 \%)$, GlyT2 mRNA only $(n=4 / 33,12 \%)$, GAD67 mRNA only $(n=5 / 33,15 \%)$, or coexpression of GlyT2 and GAD67 mRNA ( $n=10 / 33,30 \%)$. Thus, $58 \%(n=19 / 33)$ of this sample of nonbursters expressed inhibitory phenotypic mRNA markers. Overall, these results show that approximately half $(n=19 / 39,49 \%)$ of pre-BötC inspiratory neurons that were identified by sampling not based on commissural axonal projection patterns are inhibitory.

\section{GAD67-GFP positive pre-BötC inspiratory neurons in transgenic mice}

We also assayed electrophysiologically identified GFP-positive neurons $(n=31)$ in the pre-BötC of GAD67-GFP transgenic mice. We used slices from these transgenic mice to increase the size of our sample of inspiratory inhibitory neurons for electrophysiological and morphological (axon projection pattern) analyses. All of the inspiratory GFP-positive neurons sampled were nonburster neurons, consistent with the results from electrophysiological recording of neurons identified by $\mathrm{Ca}^{2+}$-sensitive dye in the neonatal rat slices. These GAD67-GFP-positive nonbursters, assayed by scmRT-PCR, expressed either GAD67 mRNA only $(n=16 / 31,52 \%)$ or coexpressed GAD67 and GlyT2 mRNA $(n=14 / 31,45 \%)$. One neuron expressed GlyT2 mRNA only $(n=1 / 31,3 \%)$, and none expressed VgluT2 mRNA. Thus, we verified in GAD67-GFP mice that among the GFP-positive pre-BötC inspiratory neurons assayed, $>95 \%$ expressed GAD67 mRNA (Tamamaki et al., 2003; Ono et al., 2005) and nearly half coexpressed GAD67 and GlyT2 mRNA.

\section{Identification of glycinergic and GABAergic pre-BötC} inspiratory neurons by immunohistochemical labeling We analyzed anti-glycine immunolabeling in a set of biocytinfilled pre-BötC inspiratory neurons functionally identified after neuronal labeling by midline injection of $\mathrm{Ca}^{2+}$-sensitive dye ( $n=76$ neurons examined total; 24 intrinsic bursters, 52 nonburster inspiratory neurons). In a separate set of experiments we also analyzed anti-GABA immunolabeling $(n=42$ neurons total: 15 intrinsic bursters, 27 nonburster neurons). Figure 4 shows the overall distribution of glycine antibody-staining positive neurons $(A)$ and GABA antibody positive neurons $(B)$ in the pre-BötC region as labeled with the antibodies used, and also shows examples of a immunolabeled glycinergic $(C 1, C 2)$ and a GABAergic $(D 1, D 2)$ pre-BötC inspiratory nonburster neuron that were prelabeled with biocytin during whole-cell electrophysiological recording. None of the intrinsic bursters identified were immunopositive for either glycine antibody ( $n=24$ neurons tested) or GABA antibody $(n=15)$. Twenty-one percent of nonbursters analyzed ( $n=11 / 52)$ were positively immunolabeled with glycine antibody, and $22 \%$ of nonbursters analyzed $(n=6 / 27)$ were GABA positive. Immunostaining was not observed in somata, dendrites/neuropils, or terminals when the primary antibody was omitted as a negative control. As a further negative control, we also confirmed the absence of glycine or GABA immunolabeling in motoneurons exhibiting ChAT immunolabeling in the regions of nucleus ambiguus (NA) and XII nucleus. As a positive control, immunoreactivity of neurons located in the spinal trigeminal nucleus known to contain glycinergic and GABAergic neurons (Kuwana et al., 2006; Winter et al., 2009) or immunoreactivity of neurons located in the nucleus of Roller known to be GABAergic (van Brederode et al., 2011) was corroborated (data not shown) in every experiment. Overall, these immunolabeling results are consistent with our findings from scmRT-PCR experiments 
showing that pre-BötC intrinsic bursters with contralateral axonal projections are generally not inhibitory neurons, and that intrinsic nonbursters with such axonal projections consisted of a heterogeneous population of neurons with a small percentage (19\%) of inhibitory neurons.

We also analyzed glycine immunoreactivities of GAD67-GFP positive preBötC neurons. Nearly one-half ( $n=92$ / 188) of GAD67-GFP positive pre-BötC neurons were immunopositive for glycine (Fig. 5). This result is consistent with the scmRT-PCR results from neonatal rats that $45 \%$ of GAD67-GFP positive preBötC inspiratory neurons expressed both GAD67 and GlyT2 mRNA.

\section{Electrophysiological and biophysical} properties of glutamatergic, glycinergic, and GABAergic pre-BötC inspiratory interneurons

We compared intrinsic electrophysiological properties and spiking characteristics of glutamatergic ( $n=91$ total, 42 intrinsic bursters and 49 intrinsic nonbursters), glycinergic $(n=6)$, GABAergic $(n=5)$, and coexpressed glycinergic-GABAergic $(n=8)$ pre-BötC inspiratory neurons retrogradely labeled by midline injection of $\mathrm{Ca}^{2+}$-sensitive dye and whose neurotransmitter phenotypes were defined by subsequent scmRT-PCR assays. We also compared these commissural pre-BötC inspiratory neurons with GFP-positive pre-BötC inspiratory neurons $(n=$ 31) in GAD67-GFP transgenic mice. Table 2 summarizes biophysical parameters of these pre-BötC inspiratory neurons including whole-cell capacitance $\left(C_{\mathrm{m}}\right)$, baseline membrane potential, membrane input resistance $\left(R_{\text {input }}\right)$, and parameters of membrane conductances derived from voltage-clamp measurements of quasi-steady-state subthreshold $I-V$ relationships. Suprathreshold spiking properties including AP shape, repetitive spiking characteristics, and steady-state spiking frequency versus injected current $(f-I)$ relations were also comparatively analyzed. Note that these properties were measured at elevated extracellular $\mathrm{K}^{+}$concentrations $(8-9 \mathrm{~mm})$ that are optimal for maintaining rhythmic respiratory network activity in our slice preparations. Under these conditions, $\mathrm{K}^{+}$currents are reduced (average $E_{\mathrm{K}}=-71 \mathrm{mV}$ for our recording conditions) relative to those in vivo. Accordingly, baseline membrane potentials are more depolarized, whereas $\mathrm{K}^{+}$-dependent afterhyperpolarizations, which affect repetitive spiking behavior and AP shape, are reduced. Comparative analysis of pre-BötC inspiratory neuron properties was nevertheless possible, because all cell populations studied were exposed to similar conditions.

Passive membrane properties in pre-BötC inspiratory neurons $C_{\mathrm{m}}(\mathrm{pF})$ in glutamatergic pre-BötC inspiratory neurons (33.5 \pm 2.2 for intrinsic bursters, $34.7 \pm 2.2$ for nonbursters) (Table 2) was significantly larger than for inhibitory neurons $(29.1 \pm 2.0$ in glycinergic, $28.1 \pm 2.7$ in GABAergic, $29.3 \pm 3.4$ in glycinergicGABAergic neurons). Between intrinsic bursters and nonbursters or among inhibitory neurons, however, $C_{\mathrm{m}}$ was not significantly different. $C_{\mathrm{m}}$ in neonatal mouse GAD67-GFP positive neurons $(22.3 \pm 6.6)$ was significantly smaller than for the neonatal rat neurons, which we attribute to species difference
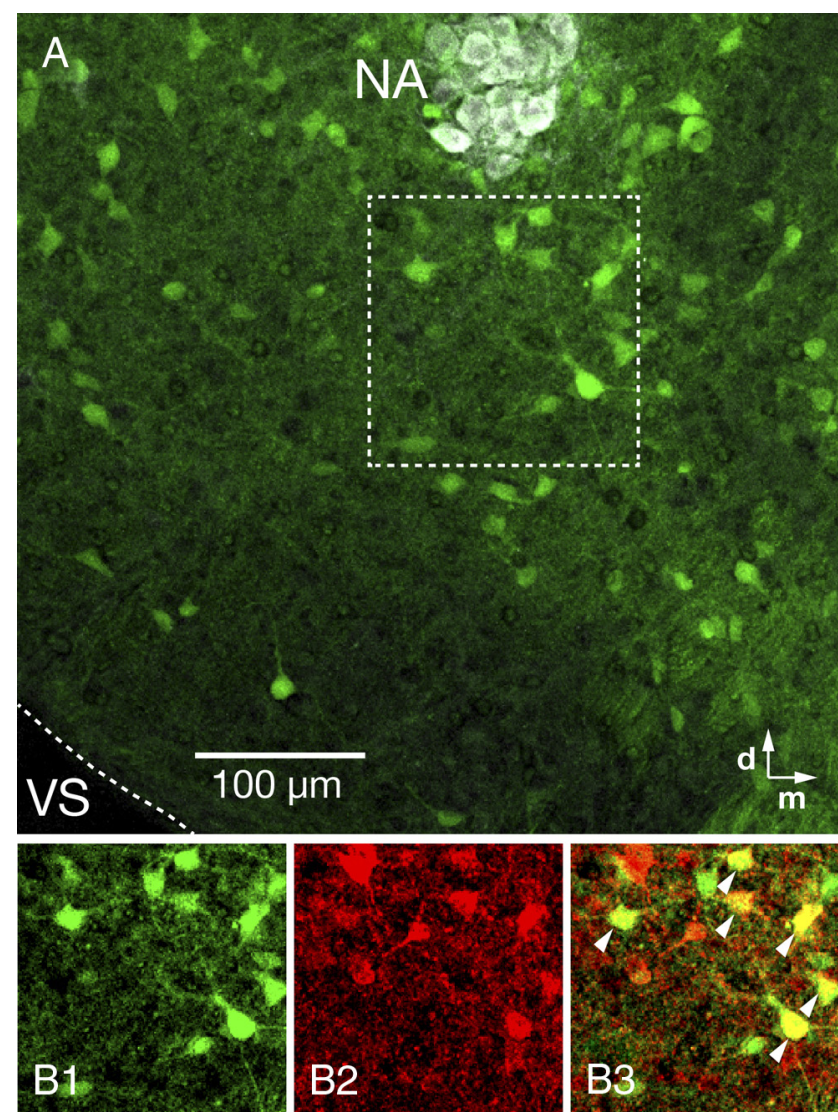

Figure 5. Distribution of GAD67-GFP positive neurons in the pre-BötC region and coexpression of glycine in GAD67-GFP transgenic mouse. A, GAD67-GFP positive neurons distributed within the pre-BötC ( $10 \times$ objective). Motoneurons in the NA were stained with ChAT antibody (white). $\boldsymbol{B}$, Single optical plane images of GAD67-GFP positive (B1) and glycine (B2) immunolabeled neurons in the pre-BötC area marked by dashed box in $\boldsymbol{A}$. Merged image (B3) shows a mixed population of pre-BötC inhibitory neurons, including GAD67-GFP positive neurons with glycine immunolabeling (yellow, arrowheads) or without glycine immunolabeling (green), and GAD67-GFP negative neurons with glycine immunolabeling (red). VS, Ventral surface. 
Table 2. Electrophysiological properties of glutamatergic, glycinergic, and GABAergic pre-BötC inspiratory neurons

\begin{tabular}{|c|c|c|c|c|c|c|}
\hline & VgluT2 $(n=42)^{a}$ & VgluT2 $(n=49)^{b}$ & GlyT2 $(n=6)^{b}$ & GAD67 $(n=5)^{b}$ & GlyT2-GAD67 $(n=12)^{b}$ & GAD67-GFP (mouse, $n=31)^{b}$ \\
\hline Baseline $V_{m}(m V)$ & $-59.0 \pm 3.2$ & $-59.6 \pm 3.4$ & $-60.8 \pm 3.6$ & $-59.9 \pm 3.1$ & $-60.6 \pm 3.6$ & $-62.8 \pm 4.0$ \\
\hline$C_{m}(p F)$ & $33.5 \pm 2.2^{*}$ & $34.7 \pm 2.2^{*}$ & $29.1 \pm 2.0$ & $28.1 \pm 2.7$ & $29.3 \pm 3.4$ & $22.3 \pm 6.6^{\dagger}$ \\
\hline$R_{\text {input }}(\mathrm{M} \Omega)$ & $298 \pm 70$ & $264 \pm 63^{*}$ & $304 \pm 52$ & $321 \pm 86$ & $315 \pm 84$ & $334 \pm 48$ \\
\hline mAHP amplitude (mV) & $3.98 \pm 0.60$ & $3.82 \pm 0.72$ & $3.88 \pm 0.65$ & $4.02 \pm 0.72$ & $4.08 \pm 0.93$ & $3.98 \pm 0.66$ \\
\hline mAHP duration (ms) & $88.0 \pm 18.9^{*}$ & $96.0 \pm 16.8$ & $104.0 \pm 18.8$ & $102.0 \pm 17.8$ & $102.3 \pm 30.2$ & $103.6 \pm 33.2$ \\
\hline$g_{\mathrm{Nap}} / C_{\mathrm{m}}(\mathrm{pS} / \mathrm{pF})$ & $91.4 \pm 14.4^{\dagger_{, *}}$ & $46.0 \pm 9.2^{*}$ & $39.6 \pm 7.1$ & $41.3 \pm 6.0$ & $38.4 \pm 12.1$ & $40.0 \pm 9.1$ \\
\hline$g_{\text {Leak }} / C_{\mathrm{m}}(\mathrm{pS} / \mathrm{pF})$ & $106.1 \pm 25.3$ & $119.5 \pm 24.3$ & $115.7 \pm 17.6$ & $117.7 \pm 33.6$ & $116.9 \pm 31.7$ & $122.8 \pm 42.1$ \\
\hline Spike frequency adaptation: decay $\tau$ & - & $1.48 \pm 0.87^{*}$ & $0.84 \pm 0.34$ & $0.79 \pm 0.22$ & $0.88 \pm 0.39$ & $0.72 \pm 0.46$ \\
\hline$F_{\text {adap }}$ index & - & $0.38 \pm 0.17^{*}$ & $0.54 \pm 0.19$ & $0.59 \pm 0.21$ & $0.52 \pm 0.17$ & $0.57 \pm 0.20$ \\
\hline$f_{0}-/$ slope $(\mathrm{Hz} / \mathrm{nA})$ & - & $188 \pm 38$ & $209 \pm 42$ & $208 \pm 37$ & $222 \pm 39$ & $228 \pm 39$ \\
\hline$f_{\mathrm{ss}}-/$ slope $(\mathrm{Hz} / \mathrm{nA})$ & - & $136 \pm 27$ & $125 \pm 26$ & $106 \pm 23$ & $130 \pm 32$ & $125 \pm 36$ \\
\hline
\end{tabular}

${ }^{\dagger}$ Significantly different from the rest; ${ }^{*}$ significantly different from inhibitory neurons (see Results for details).

${ }^{a}$ Intrinsic burster.

${ }^{b}$ Nonburster.

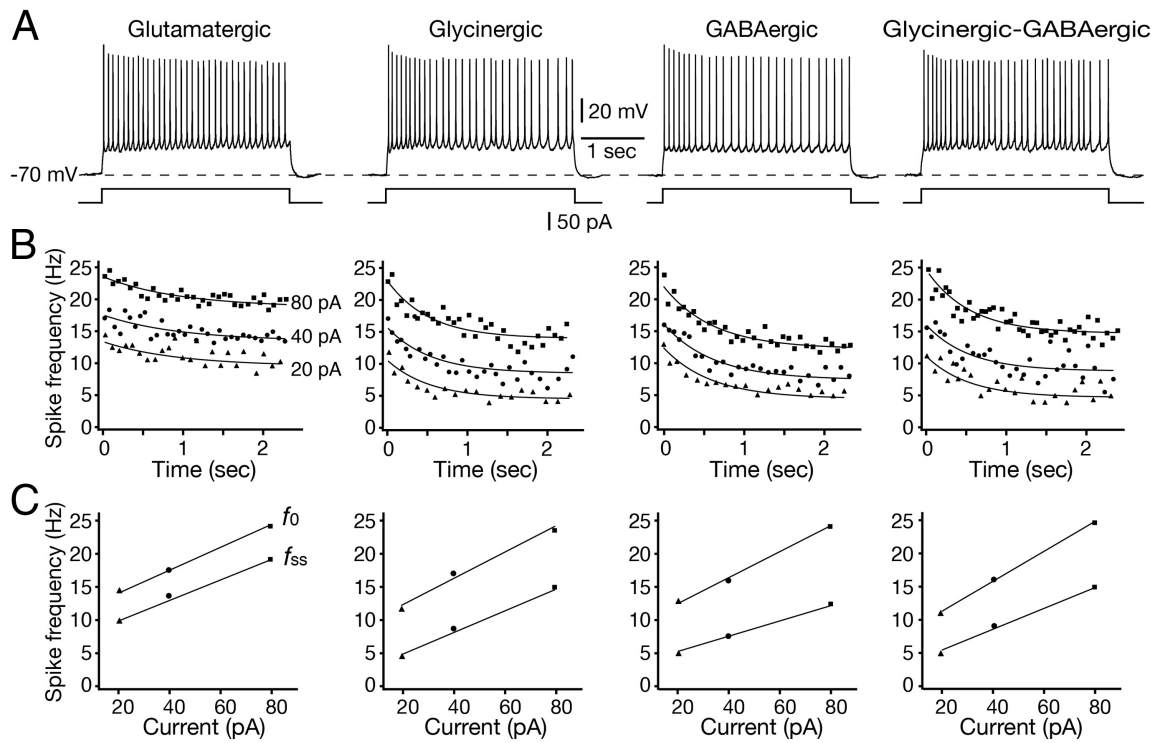

Figure 6. Repetitive firing properties of pre-BötC excitatory and inhibitory neurons during sustained depolarization. $\boldsymbol{A}$, Repetitive spiking behavior of glutamatergic, glycinergic, GABAergic, and glycinergic-GABAergic intrinsically nonburster pre-BötC inspiratory neurons. Spiking behavior was produced by sustained suprathreshold current injection (40 pA, $2.4 \mathrm{~s}$ duration) from holding membrane potentials of $-70 \mathrm{mV}$. Neurons shown were labeled retrogradely by midline injection of CaG/0GB and transmitter phenotype was identified by scmRT-PCR. $B, f-t$ profiles during repetitive spiking in all transmitter-related phenotypes of pre-BötC inspiratory neurons showed spike frequency adaptation. Spiking behaviors for three levels of sustained depolarization are shown. At all levels, the decay time constant $(\tau, s)$ derived from single exponential curve fits (solid lines) to the $f-t$ data points for glutamatergic neurons was significantly longer than that for glycinergic, GABAergic, or glycinergic/GABAergic neurons (Table 2). $\boldsymbol{C}$, Examples of $f-/$ relations for neurons shown in $\boldsymbol{A}$ and $\boldsymbol{B}$. The $f-/$ relations were analyzed for initial $f_{0}$ and $f_{\text {ss }}$ after adaptation in all cases. The slopes $(\mathrm{Hz} / \mathrm{nA})$ derived from linear fits (solid lines) to $f_{0}-l$ and $f_{s s}-/$ relationships were not statistically different for the group of cells analyzed (Table 2).

(possibly reflecting smaller neurons in mice). The $R_{\text {input }}(\mathrm{M} \Omega$ ) for the group of excitatory nonbursters $(264 \pm 63)$ was significantly smaller than for the other phenotypes of neurons $(298 \pm$ 70 for the group of intrinsic bursters, $304 \pm 52$ for glycinergic, $321 \pm 86$ for GABAergic, $315 \pm 84$ for glycinergic-GABAergic, $334 \pm 48$ for GAD67-GFP positive neurons). The baseline membrane potentials were not significantly different among all neuron types analyzed.

Action potential characteristics and repetitive spiking properties We analyzed parameters of an evoked AP shape by applying a brief ( $2 \mathrm{~ms}$ ) suprathreshold depolarizing current from the baseline membrane potential. All phenotypes of pre-BötC inspiratory neurons characteristically exhibited mAHP, but not afterdepo- larization. The durations and amplitudes of the mAHPs were not significantly different among the excitatory and inhibitory phenotypes analyzed (Table 2 ). The duration (ms) of mAHP in glutamatergic intrinsic burster neurons $(88.0 \pm 18.9)$ was significantly shorter than in the glutamatergic nonbursters $(96.0 \pm 16.8)$ and inhibitory neurons $(104.0 \pm 18.8$ in glycinergic, $102.0 \pm 17.8$ in GABAergic, $102.3 \pm 30.2$ in glycinergic-GABAergic, $103.6 \pm 33.2$ in GAD67-GFP positive preBötC inspiratory neurons). We also analyzed repetitive spiking behavior with a sustained suprathreshold current injection (20, 40, $80 \mathrm{pA}, 2.4 \mathrm{~s})$ from a holding membrane potential of $-70 \mathrm{mV}$. The spike frequency versus time $(f-t)$ profiles during repetitive spiking in all phenotypes of pre-BötC inspiratory neurons analyzed showed spike frequency adaptation (Fig. $6)$. The decay time constant $(\tau, s)$ derived from single exponential fits to the $f-t$ relations for glutamatergic nonburster neurons $(1.48 \pm 0.87)$ was significantly longer than that for glycinergic $(0.84 \pm 0.34)$, GABAergic (0.79 \pm 0.22$)$, glycinergicGABAergic $(0.88 \pm 0.39)$, or GAD67-GFP positive $(0.72 \pm 0.46)$ pre-BötC nonburster inspiratory neurons. Spike frequency adaptation index $\left(F_{\text {adap }}\right)\left[F_{\text {adap }}=\right.$ $\left(f_{0}-f_{\mathrm{ss}}\right) / f_{0}$, where $f_{0}$ is the initial spike frequency, $f_{s s}$ is the steady-state spike frequency] of glutamatergic nonburster neurons $(0.38 \pm 0.17)$ was also significantly smaller than that of glycinergic $(0.54 \pm 0.19)$, GABAergic $(0.59 \pm 0.21)$, glycinergic-GABAergic $(0.52 \pm 0.17)$, or GAD67-GFP positive $(0.57 \pm 0.20)$ pre-BötC nonburster neurons. These results indicated that inhibitory pre-BötC inspiratory neurons showed earlier and stronger spike frequency adaptation than excitatory neurons. The $f-I$ relations were analyzed for initial spike frequency $\left(f_{0}\right)$ and steady-state spike frequency $\left(f_{s s}\right)$. The slopes $(\mathrm{Hz} / \mathrm{nA})$ from linear fits to the $f_{0}-I$ relationships were $188 \pm 38$ for glutamatergic, $209 \pm 42$ for glycinergic, $208 \pm 37$ for GABAergic, $222 \pm 39$ for glycinergic-GABAergic, and $228 \pm 39$ for GAD67-GFP positive pre-BötC inspiratory neurons, which were not statistically different $(p>0.05)$ for the groups of cells 
analyzed. The slopes from the linear fits to $f_{s s}-I$ relation were $136 \pm 27$ for glutamatergic, $125 \pm 26$ for glycinergic, $106 \pm 23$ for GABAergic, $130 \pm 32$ for glycinergic/GABAergic, and $125 \pm 36$ for GAD67-GFP positive pre-BötC inspiratory neurons, which were not statistically different $(p>0.05)$.

Responses of pre-BötC inspiratory neurons to hyperpolarization We examined voltage responses of pre-BötC inspiratory neurons to hyperpolarization because the hyperpolarization-activated current $I_{\mathrm{h}}$ has been implicated in intrinsic rhythmic bursting properties of respiratory neurons and is thought to play an important modulatory role in respiratory rhythm generation in vitro (Thoby-Brisson et al., 2000, 2009). Under current-clamp recording, in response to a series of hyperpolarizing current injections from the baseline membrane potential, some inhibitory inspiratory neurons $(n=8 / 19,42 \%)$ and a small fraction of the excitatory nonburster neurons tested $(n=7 / 91,8 \%)$ displayed a depolarizing sag. At larger amplitudes of hyperpolarizing current pulses, the amplitude of the sag potential increased (Fig. 7A), indicating an $I_{\mathrm{h}}$-like conductance. The other pre-BötC inspiratory neurons exhibited only passive membrane responses to hyperpolarizing current pulses (Fig. $7 B$ ). We then measured the amplitude of the hyperpolarization-activated currents from the difference between the instantaneous current immediately following the capacitative transient and the steady-state current measured at the end of the $1 \mathrm{~s}$ hyperpolarizing voltage steps (from $-65 \mathrm{mV}$ to -120 $\mathrm{mV}$ ) under voltage-clamp (Fig. 7C). The amplitude of $I_{\mathrm{h}}$ was between -80 and $-240 \mathrm{pA}$ (mean $-146 \mathrm{pA}, n=15$ ) in pre-BötC inspiratory neurons that exhibited the sag response in current-clamp recordings.

Subthreshold membrane properties

Subthreshold $I-V$ relations for post hoc identified excitatory or inhibitory pre-BötC inspiratory neurons were evaluated from slow voltage-clamp ramps $(30 \mathrm{mV} / \mathrm{s} ;-110$ to $+10 \mathrm{mV})$. All neurons exhibited non-monotonic, $\mathrm{N}$-shaped $I-V$ relations which decompose into two primary subthreshold conductances: an ohmic-like voltage-insensitive leak conductance characterized by the essentially linear region of the $I-V$ curve below $-65 \mathrm{mV}$, and a voltage-activated, TTX-sensitive, inward persistent $\mathrm{Na}^{+}$ current generating the negative slope region in the $-60 \mathrm{mV}$ to $-40 \mathrm{mV}$ membrane voltage range. Leak conductance $\left(g_{\text {Leak }}\right)$ was determined by linear regression to the slope of the passive region $(-110$ to $-65 \mathrm{mV})$ of the $I-V$ relation. The total leak current in all neurons examined had a reversal potential $E_{\text {Leak }}$ between -70 and $-60 \mathrm{mV}$ (mean $E_{\text {Leak }}=-68 \pm 3.2 \mathrm{mV}$ ), similar to the potential previously determined for $E_{\text {Leak }}$ (Koizumi and Smith, 2008). The values of conductance densities for $I_{\text {Leak }}\left(g_{\text {Leak }} / C_{\mathrm{m}}\right.$, $\mathrm{pS} / \mathrm{pF}$ ) (Table 2) were not significantly different among glutamatergic intrinsic bursters $(106.1 \pm 25.3)$, glutamatergic nonbursters (119.5 \pm 24.3$)$, glycinergic (115.7 \pm 17.6), GABAergic (117.7 \pm 33.6), glycinergic-GABAergic (116.9 \pm 31.7), and GAD67-GFP positive (122.8 \pm 42.1$)$ pre-BötC inspiratory neurons. Subtraction of $I_{\text {Leak }}$ from the total $I-V$ curve revealed the subthreshold voltage-activated current components generating the negative slope region, which was primarily attributable to a TTX-sensitive, subthreshold-activating persistent $\mathrm{Na}^{+}$current $\left(I_{\mathrm{NaP}}\right)$ as we have previously documented for pre-BötC inspiratory neurons (Koizumi and Smith, 2008). We extracted the TTXsensitive $I_{\mathrm{NaP}}$ component by subtraction of the total $I-V$ curves obtained before and after TTX. We calculated the peak amplitude of $I_{\mathrm{NaP}}(90-260 \mathrm{pA})$ from the difference in $I-V$ relationships and compared $I_{\mathrm{NaP}}$ conductances of glutamatergic, glycinergic, and

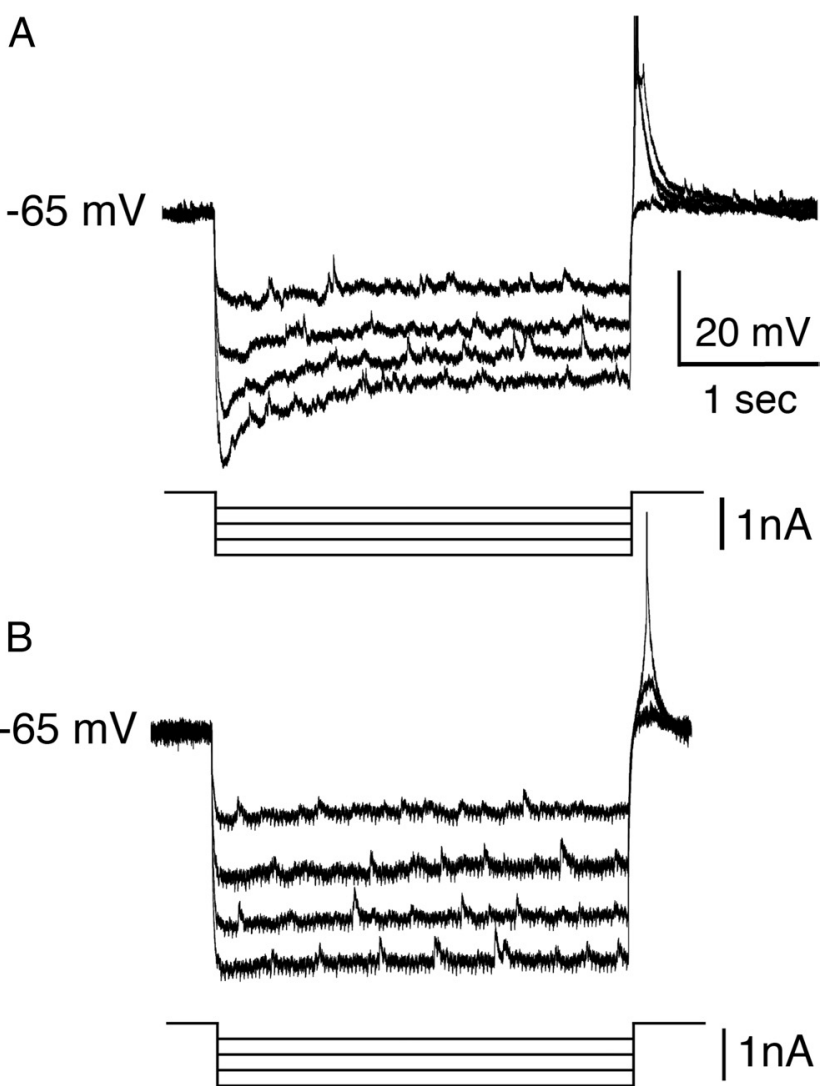

C

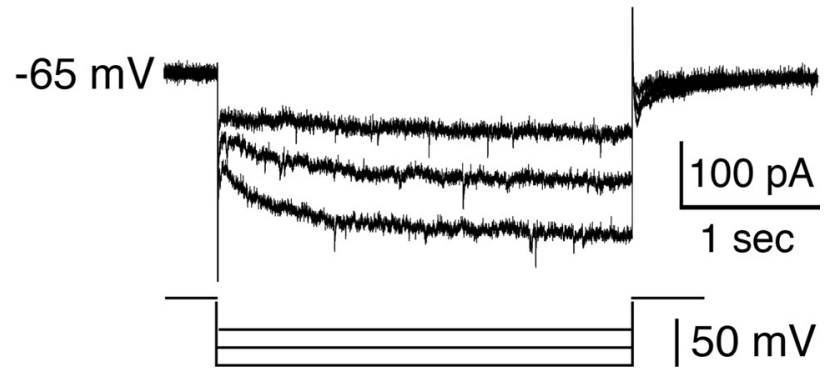

Figure 7. Hyperpolarization-activated currents in pre-BötC inspiratory neurons. $A$, Under current-clamp recording, in response to a series of hyperpolarizing current pulses from a membrane potential of $-65 \mathrm{mV}$, this glycinergic pre-BötC inspiratory neuron identified by scmRTPCR displayed an initial sag and a posthyperpolarization rebound depolarization. At larger amplitudes of hyperpolarizing current pulses, the amplitude of the sag response increased. $\boldsymbol{B}$ Example of an identified glutamatergic pre-BötC inspiratory neuron exhibiting only passive membrane responses to hyperpolarizing current pulses under current-clamp recording. C, Example of voltage-clamp recordings from a glycinergic pre-BötC inspiratory neuron, illustrating hyperpolarization-activated currents defined by the difference between the instantaneous inward current immediately following the capacitative transient and steady-state current, which was measured at the end of the 1 s hyperpolarizing voltage steps (from $-65 \mathrm{mV}$ to $-120 \mathrm{mV}$ ).

GABAergic pre-BötC inspiratory neurons. Estimated $I_{\mathrm{NaP}}$ maximal conductance densities $\left(g_{\mathrm{NaP}} / C_{\mathrm{m}}, \mathrm{pS} / \mathrm{pF}\right.$ ) (Table 2) for glutamatergic intrinsic bursters $(91.4 \pm 14.4)$ and nonbursters $(46.0 \pm 9.2)$ were significantly higher than that for glycinergic $(39.6 \pm 7.1)$, GABAergic (41.3 \pm 6.0$)$, glycinergic-GABAergic $(38.4 \pm 12.1)$, and GAD67-GFP positive $(40.0 \pm 9.1)$ inhibitory inspiratory neurons. We also compared glutamatergic intrinsic bursters $(n=44)$ and glutamatergic nonbursters $(n=49)$, and 
A
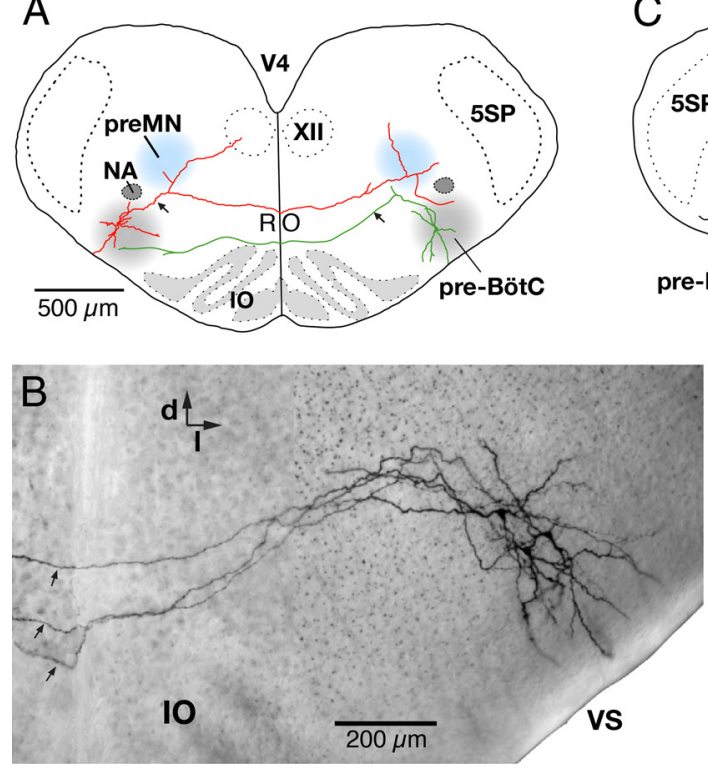
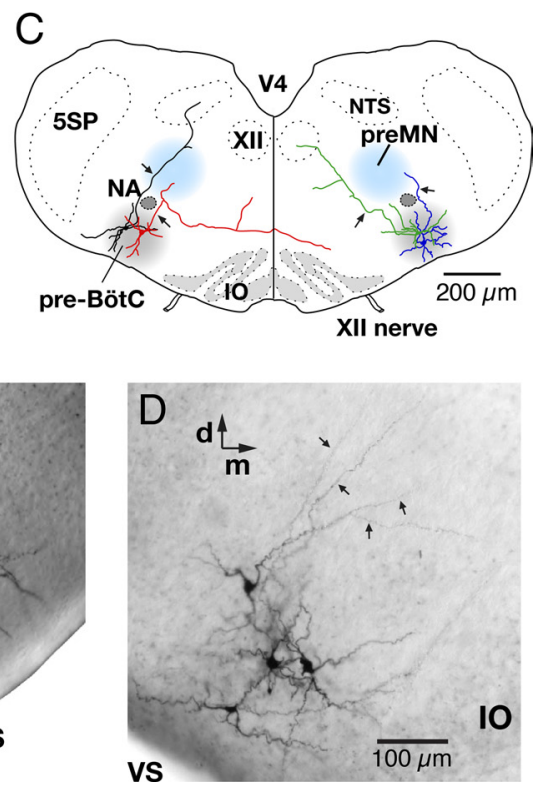

Figure 8. Axonal projection patterns of pre-BötC inspiratory neurons. $A$, Representative examples of axonal projection patterns of pre-BötC inspiratory neurons retrogradely labeled by midline injection of $\mathrm{Ca}^{2+}$-sensitive dye. An identified pre-BötC intrinsic burster neuron (red) with axon (arrow) collaterals projecting through the ipsilateral inspiratory XII preMN area dorsomedial to the $\mathrm{NA}$, ipsilateral XII motor nucleus, and the contralateral pre-BötC. This neuron also had collateral projections to the contralateral preMN area. Example of a pre-BötC nonburster neuron (green) with axonal projections to the contralateral pre-BötC and toward the ipsilateral XII preMN region. B, Composite, multifocal, infrared, bright-field CCD image showing the dendritic arborizations and commissural axonal projections of 3 pre-BötC inspiratory neurons filled with biocytin visualized in a whole-mount slice preparation. The axons (arrows) projected all the way to the contralateral pre-BötC (data not shown). C, Representative examples of reconstructed axonal projection patterns of GAD67-GFP positive pre-BötC inspiratory neurons (nonbursters) in GAD67-GFP transgenic mice. One of the neurons shown (red) had an axon (arrow) that projected through the ipsilateral preMN area and to the contralateral side. Another neuron (green) had an axon (arrow) with projections ipsilaterally to theXII motor nucleus through the ipsilateral preMN area. The other GFP-positive neuron (black) illustrated had axonal projections ipsilaterally through the preMN area to the region of the NTS. D, Bright-field image (compare B) of four GAD67-GFP positive pre-BötC inspiratoryneurons (nonbursters) showing axons (arrows) projecting dorsally through the pre-BötC toward the ipsilateral preMN zone. $5 S P$, Trigeminal spinal nucleus; 10 , inferior olivary nucleus; $\mathrm{RO}$, raphe obscurus nucleus; VS, ventral surface; $\mathrm{V}$, fourth ventricle; $d$, dorsal; $m$, medial; I, lateral.

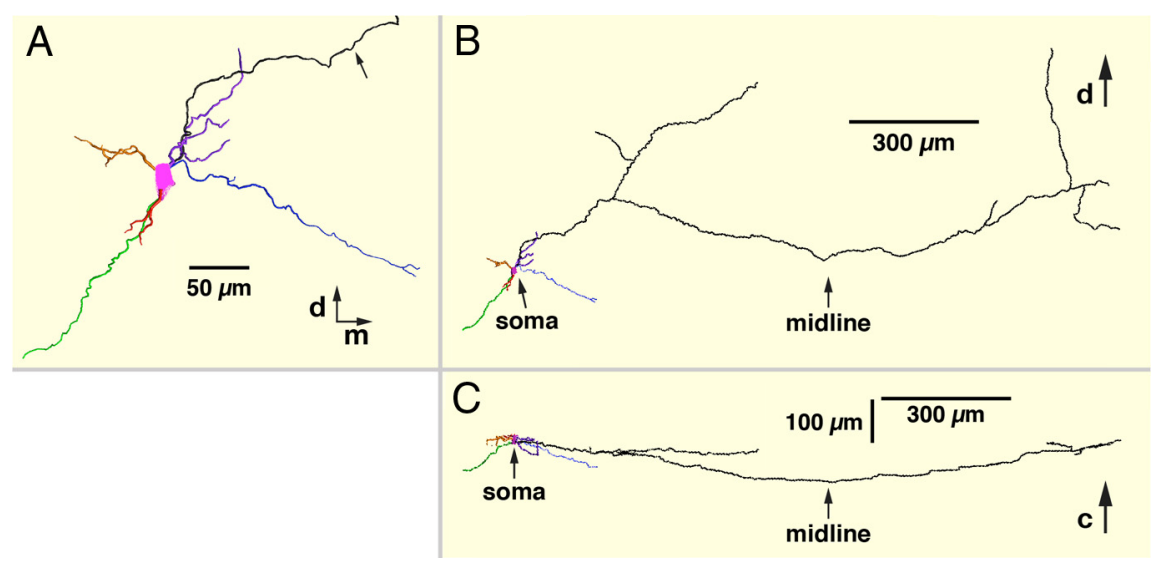

Figure 9. 3D reconstruction of dendritic arborization and axonal projections of a pre-BötC intrinsic burster neuron. $A, X Y$ (coronal) plane view (caudal end of slice) of reconstructed (whole-mount) dendritic arborizations of pre-BötC inspiratory intrinsic burster neuron (red neuron from Fig. $8 \mathrm{~A}$ ). Arrow points to neuron axon. Dorsal $(\mathrm{d})$ and medial $(\mathrm{m})$ directions are indicated. $\boldsymbol{B}, \mathrm{XY}$ plane view of axonal projection of neuron in $\boldsymbol{A}$ showing collateral axon projections to the ipsilateral preMN region and XII motor nucleus and into the contralateral pre-BötC and XII preMN regions. Location of the slice midline is indicated. C, XZ plane (ventral side) view of the axonal projection of same neuron as $\boldsymbol{B}$ illustrating the rostral (downward) trajectory of the axon as it approaches and crosses the midline before coursing caudally (upward) to approach the plane of origin at the contralateral pre-BötC.

found that the $g_{\mathrm{NaP}} / C_{\mathrm{m}}$ value of intrinsic bursters $(91.4 \pm 14.4)$ was significantly higher than that of nonbursters (46.0 \pm 9.2$)$, as predicted by our previous studies (Koizumi et al., 2008).
Axonal projection patterns of pre-BötC inspiratory neurons

We analyzed the axonal projection patterns of biocytin-filled intrinsic burster $(n=8)$ and nonburster $(n=14)$ inspiratory neurons labeled by midline injection of $\mathrm{Ca}^{2+}$-sensitive dyes (Figs. 8A,B, 9). Axonal projections of these neurons were reconstructed from whole-mount preparations of the slice (Fig. $8 B, D$ ). Axons were identified as smaller diameter processes that typically arose proximally from a large-diameter primary dendrite and could be traced for distances $>500 \mu \mathrm{m}$ beyond the dendritic field. Axonal projection patterns for this group of neurons are summarized in Table 3. All pre-BötC inspiratory neurons analyzed $(n=22)$ in the whole-mounts had commissural axons projecting into the contralateral preBötC. This confirmed that our midline injection and retrograde $\mathrm{Ca}^{2+}$-sensitive dye loading approach reliably labels commissural inspiratory neurons with axons projecting all the way to the contralateral pre-BötC, which had not specifically been verified, although it is also possible that a small fraction of the retrogradely labeled neurons were VRC respiratory neurons that project to the medullary raphe (Nuding et al., 2009). Individual axons typically coursed rostrally as they approached and crossed the midline, but then coursed back caudally as they approached and entered the contralateral pre-BötC. The rostral-caudal deviation of the axonal location through the entire trajectory of the projection was typically $<100 \mu \mathrm{m}$ within the thickness of the slice (Fig. 9). Thus, overall, these commissural axonal projections were nearly planar. Axons projected preferentially to either the ventral (Fig. $8 A$, green neuron) or dorsal (Fig. $8 A$, red neuron) zones of the contralateral pre-BötC region (Fig. $8 \mathrm{~A}$, gray shaded area), where axons entering and appearing to terminate in the ventral half or dorsal half of this area were classified (Table 3), respectively, as having ventral axonal trajectories or dorsal trajectories, with potential implications for local integration of synaptic inputs.

The axons of most pre-BötC inspiratory neurons $(n=8 / 8,100 \%$ for intrinsic bursters and $n=12 / 14,86 \%$ for intrinsic nonbursters) also projected through the ipsilateral reticular formation area containing inspiratory XII premotoneurons (preMNs), which are primarily arrayed dorsomedial to the nucleus ambiguus as previously described (Koizumi et al., 2008), and these axons had locally branching collaterals with fine arborizations in the area. The other axons coursed more ventrally before crossing the mid- 


\begin{tabular}{|c|c|c|c|c|}
\hline \multirow[b]{2}{*}{ Neuron type $(n=46)$} & \multicolumn{4}{|l|}{ Projection } \\
\hline & $\begin{array}{l}\text { Contralateral } \\
\text { pre-BötC }\end{array}$ & $\begin{array}{l}\text { Ipsilateral } \\
\text { preMN }\end{array}$ & $\begin{array}{l}\text { Contralateral } \\
\text { preMN }\end{array}$ & $\begin{array}{l}\text { Ipsilateral } \\
\text { XII motor }\end{array}$ \\
\hline Intrinsic burster $(n=8)$ & $8^{a}(100 \%)$ & $8(100 \%)$ & $2(25 \%)$ & $1(13 \%)$ \\
\hline Nonburster $(n=14)$ & $14^{b}(100 \%)$ & $12(86 \%)$ & $4(29 \%)$ & $4(29 \%)$ \\
\hline $\begin{array}{l}\text { Nonburster (GAD67-GFP) } \\
\qquad(n=24)\end{array}$ & $0(0 \%)$ & $13(54 \%)$ & $0(0 \%)$ & $8(33 \%)$ \\
\hline
\end{tabular}

${ }^{a} 2$ dorsal, 6 ventral trajectories.

${ }^{b} 5$ dorsal, 9 ventral trajectories (see Results).

line ( $n=2 / 14,14 \%$ for intrinsic nonbursters). Some pre-BötC inspiratory neurons $(n=1 / 8,25 \%$ for intrinsic bursters and $n=$ $4 / 14,29 \%$ for nonbursters) had ipsilateral axonal projections to the XII motor nucleus with branching arborization within the nucleus. A few pre-BötC inspiratory neurons $(n=2 / 8,25 \%$ for intrinsic bursters and $n=4 / 14,29 \%$ for nonbursters) had axonal projections into the contralateral preMN region (Figs. $8 A, 9$ ). No axonal projections to the contralateral XII motor nucleus were found. We also observed fine axonal processes and punctate-like endings (presumably axon terminals) in the pre-BötC and XII preMN regions. We note that while we had identified electrophysiological phenotypes of these neurons, we were unable to run scmRT-PCR assays for these neurons because cytoplasmic harvesting destroyed neuronal somas and often resulted in leakage of dye that interfered with complete reconstruction of neuronal processes. Considering that all intrinsic bursters and the majority of nonbursters identified by retrograde labeling were glutamatergic, it is likely that the commissural and axon collateral projection patterns described above are representative of glutamatergic neurons.

GAD67-GFP positive pre-BötC inspiratory neurons

We analyzed axonal projection patterns of biocytin-filled GAD67-GFP positive pre-BötC inspiratory neurons $(n=24$, Table 3 ) in whole-mounts of the slices from the GAD67-GFP transgenic mice (Figs. $8 C, D$ ). Only one GFP-positive pre-BötC neuron from this group had an axon that crossed the midline and projected into the contralateral ventrolateral reticular formation (Fig. $8 C$ ), but without reaching the contralateral pre-BötC. The axons of these inhibitory inspiratory neurons frequently projected into the ipsilateral inspiratory XII premotoneuron area (Fig. $8 C, D)(n=13 / 24,54 \%)$. A subset of these pre-BötC inhibitory neurons $(n=8 / 24,33 \%)$ had ipsilateral axonal projections to the XII motor nucleus (Table 3 ), and some ( $n=5 / 24,21 \%$ ) had axonal projections to the ipsilateral nucleus tractus solitarii (NTS) region (Fig. 8C).

\section{Dendritic morphology of excitatory and inhibitory pre-BötC inspiratory neurons}

We completely reconstructed and compared 3D morphological features of dendrites of a representative set of neonatal rat excitatory ( $n=16$ total, 3 intrinsic bursters, 13 nonbursters), and inhibitory ( $n=12$ total, 3 glycinergic, 5 GABAergic, 4 coexpressing glycinergic-GABAergic) pre-BötC inspiratory neurons filled with Alexa 594 dyes during whole-cell recordings in the rat slice preparations. All of these reconstructed neurons were from the group of cells that were imaged live in situ and their neurotransmitter phenotypes were subsequently identified by scmRTPCR assay. Representative 3D morphological reconstructions of excitatory (VgluT2 mRNA-expressing) pre-BötC inspiratory neurons including intrinsic bursters are shown in Figures 9 and 10, and for inhibitory neurons in Figure 11.

Dendritic projection ranges of pre-BötC inspiratory neurons In all pre-BötC inspiratory neurons imaged live and reconstructed, dendrites extended beyond the zone of pre-BötC neuron somata as mapped by the $\mathrm{Ca}^{2+}$ fluorescence imaging and superimposed Dodt contrast structural images. All of these neurons were multipolar, with dendritic projections extending ventrally close to the ventral medullary surface and dorsally toward the XII preMN region, similar to that presented in Figure 8. Sholl analysis in Neurolucida Explorer software was used to quantify and compare size-related parameters in dendrites of excitatory and inhibitory inspiratory neurons. Preliminary analyses did not reveal differences in the morphometrics between excitatory intrinsic bursters and nonbursters, or within the group of inhibitory neurons (glycinergic, GABAergic, coexpressing glycinergic-GABAergic). We therefore separately pooled excitatory and inhibitory neurons for the final morphometric analyses.

Dendritic size-related parameters (Li et al., 2005) analyzed were maximum dendritic projection radii (MaxProjectRadii), maximum mediolateral dendritic projection distance $(M a x M L)$, maximum dorsoventral projection distance $($ MaxDV), maximum rostrocaudal projection distance $(\operatorname{Max} R C)$, average Euclidian distance (EucDist), maximum path distance (PathDist), and cluster (Table 4). MaxML, MaxDV, MaxRC, and MaxProjectRadii were dendritic-length based parameters that considered the longest dendritic projection in the given situation. MaxProjectRadii and MaxDV of excitatory pre-BötC neurons were significantly longer than those of inhibitory neurons (Table 4). The average ratio of MaxRC/MaxDV was 0.27 and 0.40 for excitatory and inhibitory neurons, respectively, indicating compactness of both types of neurons in the rostrocaudal plane (Figs. 10, 11). EucDist and PathDist were dendritic-length based parameters that considered all branches in the dendritic arborization. EucDist and PathDist of excitatory neurons were significantly longer than inhibitory neurons. Cluster, the lowest Sholl sphere radii to include $80 \%$ of the dendritic surface area, was analyzed to indicate the availability of space, potentially for synaptic inputs, as compared with the dendritic-length based measures. Cluster in excitatory neurons was significantly (42\%) larger than in inhibitory neurons. Overall, excitatory pre-BötC inspiratory neurons exhibited $38 \%-82 \%$ larger dendritic distances than the inhibitory neurons, except for MaxML and $\operatorname{MaxRC}$, which were not significantly different.

Together, these results suggest that the dendritic arborizations of both excitatory and inhibitory interneurons are arrayed asymmetrically and project primarily in the coronal plane. However, dendritic projections of excitatory pre-BötC inspiratory neurons were longer than those of inhibitory neurons in the coronal plane. Therefore, inhibitory neurons are spatially more compact.

\section{Dendritic arborization of pre-BötC inspiratory neurons}

We also quantified and compared dendritic arborization-related parameters of reconstructed pre-BötC inspiratory neurons: total (cumulative) dendritic length ( $\Sigma$ DLength), spatially averaged dendritic diameter (AvgDiam), total dendritic surface area ( $\Sigma$ DSurfArea), and total dendritic volume ( $\Sigma$ DVolume) (all cumulative measures obtained from Sholl analysis). Using L-Measure software, we analyzed number of primary dendrites (NumPDendrite), number of total branches (NumBranch), number 


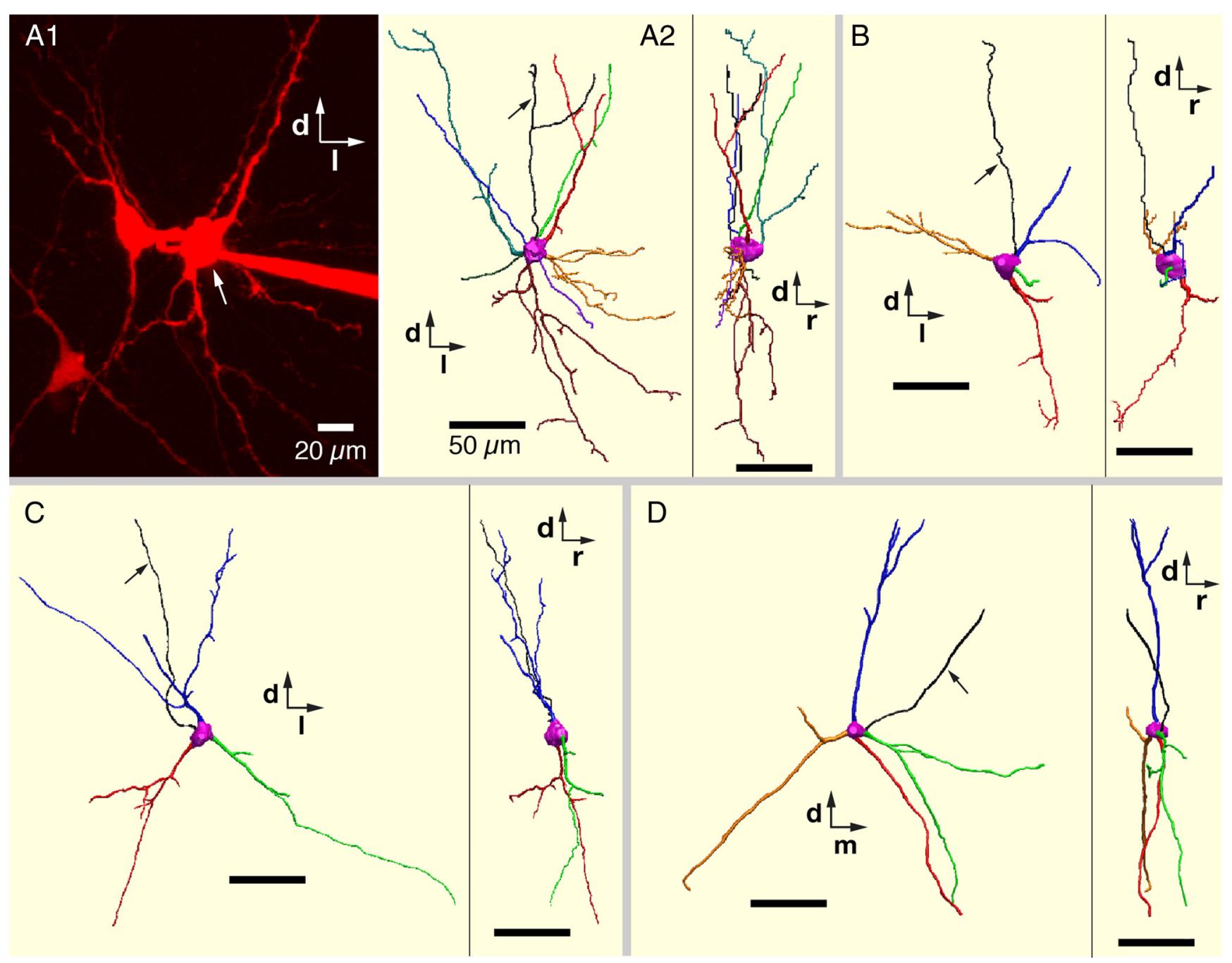

Figure 10. 3D reconstructions of dendritic arborizations of excitatory pre-BötC inspiratory neurons. A1, Two-photon live image (Z-projection of optical sections) of pre-BötC inspiratory intrinsic burster neuron (arrow) in vitro labeled with Alexa 594 dye. The two adjacent labeled neurons are pre-BötC inspiratory intrinsic nonburster neurons. Dorsal (d) and lateral (I) directions are indicated. A2, 3D reconstruction shows XY view (caudal view) and YZ view (right side parasagittal view) of the intrinsic burster neuron in $\boldsymbol{A} \boldsymbol{1}$ (arrow). Rostral (r) direction is indicated. $\boldsymbol{B}$, Another example of 3D reconstruction (caudal and right-side views) of a pre-BötC inspiratory intrinsic burster neuron. $C, D, 3$ D reconstructions (caudal and side views) of two glutamatergic pre-BötC inspiratory nonburster neurons. Arrows in $\boldsymbol{A} \mathbf{2}, \boldsymbol{B}-\boldsymbol{D}$ point to axons. Scale bars: $50 \mu \mathrm{m}$.

of bifurcations (NumBif), number of terminations (NumTerm), and maximum branch order (MaxBO) (Table 5). ¿DLength and ¿DSurfArea in excitatory neurons were significantly larger (by $82 \%$ and $81 \%$, respectively) than in inhibitory neurons. NumBranch, NumBif, and NumTerm of excitatory neurons indicated $53 \%$ more branches, $63 \%$ more bifurcations, and $53 \%$ more terminations than inhibitory neurons (all statistically significant). These results suggest that although excitatory and inhibitory neurons have nearly the same number of primary dendrites (NumPDendrite was not significantly different), in the same spatial area, excitatory neurons have more net dendritic material. This may be reflected in the larger membrane capacitance values of the excitatory neurons (Table 2), which may also reflect larger soma size of these cells although we did not explicitly measure soma dimensions because of possible distortion of soma shapes due to attachment of the whole-cell recording pipette.

In summary, our morphometric analyses provide strong evidence that not only are the dendritic projection ranges larger but also that the dendritic arborization in a given spatial area is significantly more complex in excitatory pre-BötC inspiratory neurons.

\section{Dendritic spines}

We previously reported prominent dendritic spines in a subset of pre-BötC neurons (Koizumi et al., 2008), but the transmitterrelated phenotypes of these neurons were unknown. In the present set of morphometrically analyzed neurons, we found that subsets of both excitatory $(n=11 / 16,69 \%)$ and inhibitory $(n=$ 4/12, 33\%) pre-BötC inspiratory neurons exhibited prominent spines on proximal dendrites (Fig. 12), which may represent sites of excitatory synaptic connections (Yuste and Denk, 1995; Nimchinsky et al., 2002).

\section{Discussion}

The pre-BötC has long been identified as the VRC structure containing microcircuits critical for respiratory rhythm generation (Smith et al., 1991; Feldman and Del Negro, 2006). Precisely how structural-functional properties of excitatory and inhibitory interneurons reflect or define the rhythmogenic function of the pre-BötC has not been determined.

We discovered previously undifferentiated structuralfunctional features of excitatory versus inhibitory interneurons: (1) contralaterally projecting pre-BötC inspiratory 
interneurons are primarily glutamatergic; (2) a subset of these commissural excitatory neurons have intrinsic oscillatory bursting properties; (3) commissural excitatory neurons havedivergent axonal projections to premotoneuron and motoneuron areas; (4) distinct subpopulations of glycinergic, GABAergic, and glycine-GABA interneurons exist; (5) many inhibitory neurons have ipsilateral axonal collateral projections, but only a minority have local commissural projections; (6) inhibitory neurons generally do not have intrinsic oscillatory bursting properties; (7) inhibitory interneurons are also distinguished electrophysiologically by their more pronounced spike frequency adaptation properties; (8) dendrites of both excitatory and inhibitory neurons arborize primarily in the coronal plane; and (9) dendritic fields of inhibitory neurons are locally more compact.

\section{Glutamatergic commissural} neurons are a major component of pre-BötC circuitry

Commissural pre-BötC inspiratory neurons were largely (81\%) glutamatergic. Our probing for mRNA expression identified glutamatergic neurons with high fidelity: no VgluT2-expressing neurons expressed GAD67 or GlyT2 mRNA.

Glutamatergic pre-BötC neurons have previously been identified by expression of neurokinin type1 receptor (NK1R), somatostatin (SST), or transcription factor Dbx1, but these markers are indirect and not exclusive. NK1Rexpressing neurons were mainly glutamatergic from in situ hybridization for VgluT2 mRNA (Guyenet et al., 2002); 40\% of these cells projected to the contralateral pre-BötC (Wang et al., 2001). Most (>90\%) pre-BötC SST-expressing neurons were glutamatergic by in situ hybridization for VgluT2 mRNA, and $75 \%$ of these cells had contralateral projections (Stornetta et al., 2003). Ontogenetic studies (Bouvier et al., 2010; Gray et al., 2010) revealed that most pre-BötC Dbx1-derived neurons express VgluT2 mRNA, including subsets of NK1R- and SST-expressing neurons. Bouvier et al. (2010) have also concluded that contralateral connection formation is a fundamental property of a critical subset of Dbx1-expressing pre-BötC glutamatergic cells. However, these and other previous studies did not identify these commissural cells as components of the respiratory network by electrophysiological measurements as we have done here. Our results establish that glutamatergic commissural inspiratory neurons are a prominent structural feature of inspiratory pre-BötC circuits, as we originally postulated (Koshiya and Smith, 1999).

\section{Distinct populations of pre-BötC inhibitory interneurons}

Pre-BötC respiratory glycinergic and GABAergic populations have previously been identified by in situ hybridization (GAD76 or GlyT2 mRNA-expression) in tissue from mature rats (Wang et al., 2001; Ezure et al., 2003) as well as in vitro slices from GlyT2GFP (Winter et al., 2009; Morgado-Valle et al., 2010) and GAD67-GFP (Kuwana et al., 2006) transgenic mice. Coexpress-
Table 4. Morphometric analyses of excitatory and inhibitory pre-BötC neuron dendrites: size-related parameters

\begin{tabular}{lccll}
\hline Parameters & Excitatory $(n=16)$ & Inhibitory $(n=12)$ & $p$ value & Excitatory/ \\
inhibitory ratio
\end{tabular}

*Significant difference.

ing glycine-GABA neurons have previously been described in rat spinal cord and medullary neurons (Spike et al., 1993; Stornetta et al., 2004). Glycine and GABA immunoreactivity has frequently been found in axon terminals (Todd et al., 1996; Saha et al., 1999), and corelease of GABA and glycine is also documented (Jonas et al., 1998; O’Brien and Berger, 1999). Our scmRT-PCR assays indicate that 53\% of inhibitory pre-BötC inspiratory neurons in neonatal rats and $45 \%$ of GFP-positive neurons in GAD67-GFP mice coexpress GlyT2 and GAD67 mRNA. It remains to be established whether this pre-BötC glycinergic-GABAergic population is developmentally transient, or represents a functionally persistent dualtransmitter population with distinct circuit connections exploiting unique properties of GABAergic and glycinergic cotransmission for inhibition in the respiratory network. 
Table 5. Morphometric analyses of excitatory and inhibitory pre-BötC neuron dendrites: arborization-related parameters

\begin{tabular}{lccll}
\hline Parameters & Excitatory $(n=16)$ & Inhibitory $(n=12)$ & $p$ value & $\begin{array}{l}\text { Excitatory/ } \\
\text { inhibitory ratio }\end{array}$ \\
\hline AvgDiam $(\mu \mathrm{m})$ & $2.07 \pm 1.07$ & $1.91 \pm 0.59$ & 0.636 & 1.08 \\
$\sum$ DLength $(\mu \mathrm{m})$ & $1229 \pm 490$ & $674.4 \pm 266.3$ & $0.001^{*}$ & 1.82 \\
$\sum$ DSurfArea $\left(\mu \mathrm{m}^{2}\right)$ & $8007 \pm 467$ & $4428 \pm 3000$ & $0.028^{*}$ & 1.81 \\
$\sum$ DVolume $\left(\mu \mathrm{m}^{3}\right)$ & $6049 \pm 6804$ & $2774 \pm 2730$ & 0.128 & 2.18 \\
MaxBO & $5.9 \pm 2.2$ & $4.3 \pm 1.7$ & 0.065 & 1.34 \\
NumBranch & $32.2 \pm 17.3$ & $20.91 \pm 8.37$ & $0.048^{*}$ & 1.53 \\
NumPDendrite & $4.4 \pm 1.6$ & $3.83 \pm 1.11$ & 0.331 & 1.14 \\
NumTerm & $19.3 \pm 9.2$ & $13.3 \pm 4.2$ & $0.047^{*}$ & 1.53 \\
NumBif & $13.9 \pm 8.1$ & $8.5 \pm 4.3$ & $0.047^{*}$ & 1.63 \\
\hline
\end{tabular}

*Significant difference.

Differential electrophysiological/biophysical properties of excitatory and inhibitory interneurons

The most unique electrophysiological feature of the identified pre-BötC excitatory neurons was their voltage-dependent intrinsic bursting properties. Nearly half of the commissural glutamatergic neurons exhibited these properties.

Consistent with previous electrophysiological results from a study using the same GAD67-GFP transgenic mouse strain (Kuwana et al., 2006), we did not find these intrinsic bursting properties in any inhibitory neurons identified by our labeling/assay methods. Another electrophysiological study (Morgado-Valle et al., 2010) identified a small population of pre-BötC glycinergic neurons with intrinsic bursting behavior in slices from GlyT2-GFP mice, although it is currently unclear from the data presented that these neurons exhibited the same voltage-dependent oscillatory bursting properties (compare Fig. 2) used as the classification criterion in our studies. More extensive sampling is required to determine whether inhibitory neurons can exhibit such voltage-dependent behavior.

The differentiating electrophysiological properties of inhibitory neurons were their significantly smaller whole-cell capacitance, larger input resistance, and more prominent spike frequency adaptation. The smaller membrane capacitance may reflect a smaller soma size and dendritic surface area of inhibitory versus excitatory neurons (below). The functional significance of the identified spike frequency adaptation property remains to be determined. This repetitive firing behavior could contribute to inspiratory phase terminating mechanisms where such adaptive properties of inhibitory neurons are postulated to play an important role (Rybak et al., 2007).

\section{Comparative morphological features of pre-BötC excitatory and inhibitory interneurons}

In addition to their commissural axonal projections, the majority of excitatory pre-BötC neurons had axon collateral projections to the ipsilateral XII preMN zone. A subpopulation also projected to the XII nucleus and/or the contralateral preMN zone. Thus, excitatory neurons generally have divergent axonal projections, which likely function for simultaneous transmission of inspiratory synaptic drive ipsilaterally and contralaterally throughout the local motor circuit within the slice. The direct excitatory connection from some pre-BötC neurons to XII motoneurons could prime XII respiratory motoneurons to initiate inspiratory activity. We note that while we found fine branching and/or punctate endings at all projection sites, suggestive of local axon terminals/synaptic con-

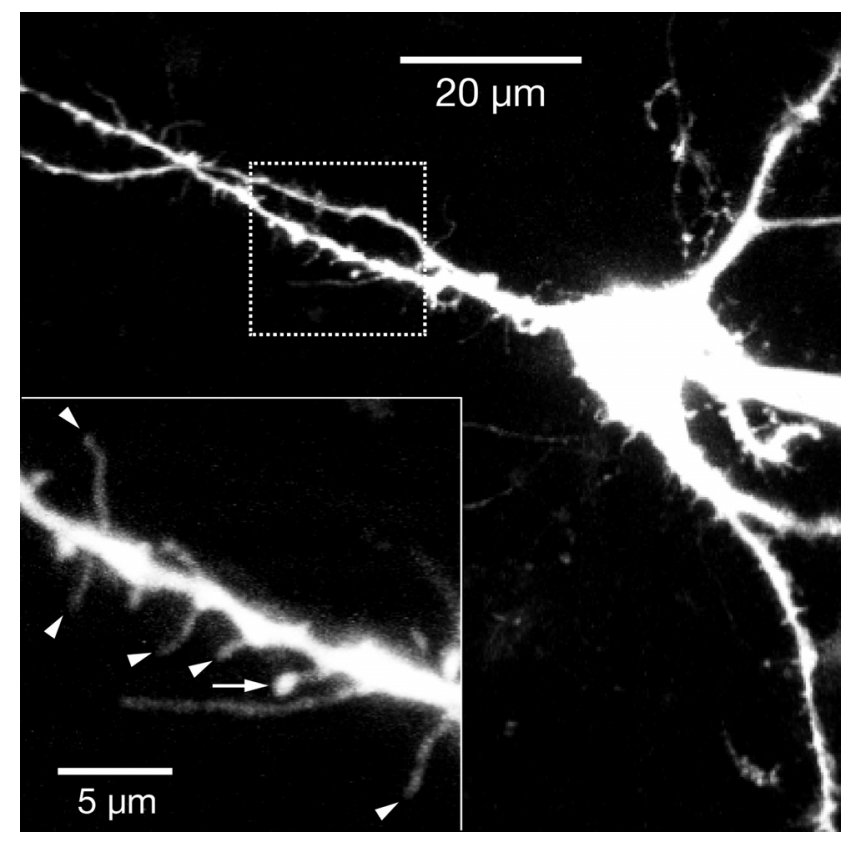

Figure 12. Dendritic spines on pre-BötC excitatory inspiratory neuron. Two-photon live Z-projection image of pre-BötC glutamatergic intrinsic burster neuron in vitro filled with Alexa 594 dye. Proximal dendrites exhibit numerous spine-like structures. Higher resolution scan image of dendritic segment (bottom left, inset, from dashed box in lower magnification image) shows examples of spines: filament-type (arrowheads) and mushroom-type (arrow pointing to spine head).

nections, we have not assayed directly for synaptic contacts (e.g., immunolabeling for synaptic proteins).

In contrast, pre-BötC inhibitory neurons exhibited mainly ipsilateral projections at the level of the slice: approximately onehalf of the inhibitory neurons projected to the ipsilateral preMN zone; a subpopulation also projected to the XII nucleus. Thus, axonal projections of inhibitory neurons are spatially organized for feedforward inhibition in the ipsilateral pre-BötC-preMNmotoneuron inspiratory drive transmission pathway. Our results, however, do not exclude inhibitory axonal projections including contralateral connections to respiratory regions not contained within the slices, since some axon collaterals may be cut at the slice surfaces.

Dendrites of both excitatory and inhibitory neurons are arrayed asymmetrically, arborizing primarily in the coronal plane. However, the dendritic projection ranges are larger and the arborization in a given spatial area is significantly more complex in excitatory neurons with implications for spatial integration of synaptic inputs.

\section{Implications for compartmental and rhythmogenic function} of pre-BötC circuits

The pre-BötC is a distinct VRC subregion with circuits functionally specialized for inspiratory rhythm generation, and it is generally recognized that excitatory subcircuits have intrinsic rhythmogenic properties (Rekling and Feldman, 1998; Koshiya and Smith, 1999; Thoby-Brisson et al., 2005). While the pre-BötC microcircuit on either side of the medulla is capable of endogenous rhythm generation (Del Negro et al., 2001; Johnson et al., 2001), bilateral synchronization by commissural glutamatergic neurons is essential for normal respiratory motor behavior (Bouvier et al., 2010). Our quantitative study provides the evidence that a substantial proportion of such commissural gluta- 
matergic neurons have intrinsic bursting properties. These properties are associated with a large membrane density of $g_{\mathrm{Nap}}$. This burst-generating conductance is ubiquitous, although with less density in nonbursters, and can function for burst synchronization as well as rhythmogenesis (Butera et al., 1999b; Purvis et al., 2007). Several studies (Ramirez et al., 2004; Del Negro et al., 2010) suggested that calcium-activated nonselective cationic current $\left(I_{\text {CAN }}\right)$ is potentially important for intrinsic or synaptically driven bursting properties of pre-BötC inspiratory neurons. It is possible that the glutamatergic intrinsic bursters identified here may also express $I_{\mathrm{CAN}}$ in addition to $I_{\mathrm{NaP}}$. Further detailed electrophysiological studies are required to ultimately determine the contributions of $I_{\mathrm{CAN}}$ to bursting in these excitatory pre-BötC neurons.

The functional role(s) of pre-BötC inhibitory interneurons have not been established. Inhibitory interactions within preBötC circuits are not obligatory for inspiratory rhythm generation in vitro (Johnson et al., 2001; Feldman and Del Negro, 2006), while these interactions are suggested to play a critical role in rhythmic pattern generation (Ramirez et al., 1997; Richter and Spyer, 2001). The inhibitory pre-BötC inspiratory neurons have been proposed to coordinate inspiratory-expiratory activity via synaptic inhibition of expiratory neurons within and outside of the pre-BötC, including in the BötC (Smith et al., 2007; Rubin et al., 2009). Furthermore, feedforward inhibition via projections to downstream inspiratory drive transmission circuits, such as to XII motoneurons and bulbospinal rostral ventral respiratory group (rVRG) circuits, may contribute to dynamic shaping of premotoneuronal inspiratory activity patterns.

A striking feature of pre-BötC inspiratory neuronal morphology is the predominantly coronal dendritic arborization of all neuron types. Moreover, contralaterally projecting axons have a nearly planar trajectory. These structural arrangements of excitatory and inhibitory pre-BötC inspiratory neurons underlie the compartmentalized organization of the pre-BötC whereby the essential contralateral and local synaptic interactions are confined within this medullary compartment and are sufficient to produce inspiratory rhythm in the bilaterally distributed preBötC oscillator.

In summary, we have comparatively differentiated electrophysiological and morphological features of pre-BötC excitatory and inhibitory interneurons. The identified features are compatible with the concepts of a compartmental organization of the pre-BötC, a bilaterally coupled excitatory rhythmogenic kernel in the pre-BötC, and a potential role of pre-BötC inhibitory neurons in shaping inspiratory pattern as well as coordinating inspiratory and expiratory activity in respiratory circuits.

\section{References}

Alheid GF, McCrimmon DR (2008) The chemical neuroanatomy of breathing. Respir Physiol Neurobiol 164:3-11. CrossRef Medline

Bouvier J, Thoby-Brisson M, Renier N, Dubreuil V, Ericson J, Champagnat J, Pierani A, Chédotal A, Fortin G (2010) Hindbrain interneurons and axon guidance signaling critical for breathing. Nat Neurosci 13:1066-1074. CrossRef Medline

Butera RJ Jr, Rinzel J, Smith JC (1999a) Models of respiratory rhythm generation in the pre-Bötzinger complex. I. Bursting pacemaker neurons. J Neurophysiol 82:382-397. Medline

Butera RJ Jr, Rinzel J, Smith JC (1999b) Models of respiratory rhythm generation in the pre-Bötzinger complex. II. Populations of coupled pacemaker neurons. J Neurophysiol 82:398-415. Medline

Del Negro CA, Johnson SM, Butera RJ, Smith JC (2001) Models of respiratory rhythm generation in the pre-Bötzinger complex. III. Experimental tests of model predictions. J Neurophysiol 86:59-74. Medline
Del Negro CA, Morgado-Valle C, Feldman JL (2002a) Respiratory rhythm: an emergent network property? Neuron 34:821-830. CrossRef Medline

Del Negro CA, Koshiya N, Butera RJ Jr, Smith JC (2002b) Persistent sodium current, membrane properties and bursting behavior of pre-Bötzinger complex inspiratory neurons in vitro. J Neurophysiol 88:2242-2250. CrossRef Medline

Del Negro CA, Hayes JA, Pace RW, Brush BR, Teruyama R, Feldman JL (2010) Synaptically activated burst-generating conductances may underlie a group-pacemaker mechanism for respiratory rhythm generation in mammals. Prog Brain Res 187:111-136. CrossRef Medline

Ezure K, Tanaka I, Kondo M (2003) Glycine is used as a transmitter by decrementing expiratory neurons of the ventrolateral medulla in the rat. J Neurosci 23:8941-8948. Medline

Feldman JL, Del Negro CA (2006) Looking for inspiration: new perspectives on respiratory rhythm. Nat Rev Neurosci 7:232-242. CrossRef Medline

Gray PA, Hayes JA, Ling GY, Llona I, Tupal S, Picardo MC, Ross SE, Hirata T, Corbin JG, Eugenín J, Del Negro CA (2010) Developmental origin of preBötzinger complex respiratory neurons. J Neurosci 30: 14883-14895. CrossRef Medline

Grillner S (2006) Biological pattern generation: the cellular and computational logic of networks in motion. Neuron 52:751-766. CrossRef Medline

Guyenet PG, Sevigny CP, Weston MC, Stornetta RL (2002) Neurokinin-1 receptor-expressing cells of the ventral respiratory group are functionally heterogeneous and predominantly glutamatergic. J Neurosci 22: 3806-3816. Medline

Johnson SM, Koshiya N, Smith JC (2001) Isolation of the kernel for respiratory rhythm generation in a novel preparation: the pre-Bötzinger complex “island." J Neurophysiol 85:1772-1776. Medline

Jonas P, Bischofberger J, Sandkühler J (1998) Corelease of two fast neurotransmitters at a central synapse. Science 281:419-424. CrossRef Medline

Koizumi H, Smith JC (2008) Persistent $\mathrm{Na}^{+}$and $\mathrm{K}^{+}$-dominated leak currents contribute to respiratory rhythm generation in the pre-Bötzinger complex in vitro. J Neurosci 28:1773-1785. CrossRef Medline

Koizumi H, Wilson CG, Wong S, Yamanishi T, Koshiya N, Smith JC (2008) Functional imaging, spatial reconstruction, and biophysical analysis of a respiratory motor circuit isolated in vitro. J Neurosci 28:2353-2365. CrossRef Medline

Koizumi H, Smerin SE, Yamanishi T, Moorjani BR, Zhang R, Smith JC (2010) TASK channels contribute to the $\mathrm{K}^{+}$-dominated leak current regulating respiratory rhythm generation in vitro. J Neurosci 30:4273-4284. CrossRef Medline

Koshiya N, Smith JC (1999) Neuronal pacemaker for breathing visualized in vitro. Nature 400:360-363. CrossRef Medline

Kuwana S, Tsunekawa N, Yanagawa Y, Okada Y, Kuribayashi J, Obata K (2006) Electrophysiological and morphological characteristics of GABAergic respiratory neurons in the mouse pre-Bötzinger complex. Eur J Neurosci 23:667-674. CrossRef Medline

Li Y, Brewer D, Burke RE, Ascoli GA (2005) Developmental changes in spinal motoneuron dendrites in neonatal mice. J Comp Neurol 483:304317. CrossRef Medline

Morgado-Valle C, Baca SM, Feldman JL (2010) Glycinergic pacemaker neurons in preBötzinger complex of neonatal mouse. J Neurosci 30:36343639. CrossRef Medline

Nimchinsky EA, Sabatini BL, Svoboda K (2002) Structure and function of dendritic spines. Ann Rev Physiol 64:313-353. CrossRef Medline

Nuding SC, Segers LS, Baekey DM, Dick TE, Solomon IC, Shannon R, Morris KF, Lindsey BG (2009) Pontine-ventral respiratory column interactions through raphe circuits detected using multi-array spike train recordings. J Neurophysiol 101:2943-2960. CrossRef Medline

O’Brien JA, Berger AJ (1999) Cotransmission of GABA and glycine to brain stem motoneurons. J Neurophysiol 82:1638-1641. Medline

Ono M, Yanagawa Y, Koyano K (2005) GABAergic neurons in inferior colliculus of the GAD67-GFP knock-in mouse: electrophysiological and morphological properties. Neurosci Res 51:475-492. CrossRef Medline

Pagliardini S, Adachi T, Ren J, Funk GD, Greer JJ (2005) Fluorescent tagging of rhythmically active respiratory neurons within the preBötzinger complex of rat medullary slice preparations. J Neurosci 25: 2591-2596. CrossRef Medline

Peña F, Parkis MA, Tryba AK, Ramirez JM (2004) Differential contribution 
of pacemaker properties to the generation of respiratory rhythms during normoxia and hypoxia. Neuron 43:105-117. CrossRef Medline

Purvis LK, Smith JC, Koizumi H, Butera RJ (2007) Intrinsic bursters increase the robustness of rhythm generation in an excitatory network. J Neurophysiol 97:1515-1526. Medline

Ramirez JM, Telgkamp P, Elsen FP, Quellmalz UJ, Richter DW (1997) Respiratory rhythm generation in mammals: synaptic and membrane properties. Respiration physiology 110:71-85. CrossRef Medline

Ramirez JM, Tryba AK, Peña F (2004) Pacemaker neurons and neuronal networks: an integrative view. Curr Opin Neurobiol 14:665-674. CrossRef Medline

Rekling JC, Feldman JL (1998) PreBötzinger complex and pacemaker neurons: hypothesized site and kernel for respiratory rhythm generation. Ann Rev Physiol 60:385-405. CrossRef Medline

Richter DW, Spyer KM (2001) Studying rhythmogenesis of breathing: comparison of in vivo and in vitro models. Trends Neurosci 24:464-472. CrossRef Medline

Rubin JE, Shevtsova NA, Ermentrout GB, Smith JC, Rybak IA (2009) Multiple rhythmic states in a model of the respiratory central pattern generator. J Neurophysiol 101:2146-2165. CrossRef Medline

Rybak IA, Abdala AP, Markin SN, Paton JF, Smith JC (2007) Spatial organization and state-dependent mechanisms for respiratory rhythm and pattern generation. Prog Brain Res 165:201-220. CrossRef Medline

Saha S, Batten TF, McWilliam PN (1999) Glycine-immunoreactive synaptic terminals in the nucleus tractus solitarii of the cat: ultrastructure and relationship to GABA-immunoreactive terminals. Synapse 33: 192-206. CrossRef Medline

Scorcioni R, Polavaram S, Ascoli GA (2008) L-Measure: a web-accessible tool for the analysis, comparison and search of digital reconstructions of neuronal morphologies. Nat Protoc 3:866-876. CrossRef Medline

Smith JC, Ellenberger HH, Ballanyi K, Richter DW, Feldman JL (1991) preBötzinger complex: a brainstem region that may generate respiratory rhythm in mammals. Science 254:726-729. CrossRef Medline

Smith JC, Butera RJ, Koshiya N, Del Negro C, Wilson CG, Johnson SM (2000) Respiratory rhythm generation in neonatal and adult mammals: the hybrid pacemaker-network model. Respir Physiol 122:131-147. CrossRef Medline

Smith JC, Abdala AP, Koizumi H, Rybak IA, Paton JF (2007) Spatial and functional architecture of the mammalian brain stem respiratory network: a hierarchy of three oscillatory mechanisms. J Neurophysiol 98: 3370-3387. CrossRef Medline

Smith JC, Abdala AP, Rybak IA, Paton JF (2009) Structural and functional architecture of respiratory networks in the mammalian brainstem. Phil Trans R Soc Lond B Biol Sci 364:2577-2587. CrossRef Medline

Spike RC, Todd AJ, Johnston HM (1993) Coexistence of NADPH diapho- rase with GABA, glycine, and acetylcholine in rat spinal cord. J Comp Neurol 335:320-333. CrossRef Medline

Stornetta RL, Rosin DL, Wang H, Sevigny CP, Weston MC, Guyenet PG (2003) A group of glutamatergic interneurons expressing high levels of both neurokinin-1 receptors and somatostatin identifies the region of the pre-Bötzinger complex. J Comp Neurol 455:499-512. CrossRef Medline

Stornetta RL, McQuiston TJ, Guyenet PG (2004) GABAergic and glycinergic presympathetic neurons of rat medulla oblongata identified by retrograde transport of pseudorabies virus and in situ hybridization. J Comp Neurol 479:257-270. CrossRef Medline

Tamamaki N, Yanagawa Y, Tomioka R, Miyazaki J, Obata K, Kaneko T (2003) Green fluorescent protein expression and colocalization with calretinin, parvalbumin, and somatostatin in the GAD67-GFP knock-in mouse. J Comp Neurol 467:60-79. CrossRef Medline

Tan W, Janczewski WA, Yang P, Shao XM, Callaway EM, Feldman JL (2008) Silencing preBötzinger complex somatostatin-expressing neurons induces persistent apnea in awake rat. Nat Neurosci 11:538-540. CrossRef Medline

Thoby-Brisson M, Telgkamp P, Ramirez JM (2000) The role of the hyperpolarization-activated current in modulating rhythmic activity in the isolated respiratory network of mice. J Neurosci 20:2994-3005. Medline

Thoby-Brisson M, Trinh JB, Champagnat J, Fortin G (2005) Emergence of the pre-Bötzinger respiratory rhythm generator in the mouse embryo. J Neurosci 25:4307-4318. CrossRef Medline

Thoby-Brisson M, Karlén M, Wu N, Charnay P, Champagnat J, Fortin G (2009) Genetic identification of an embryonic parafacial oscillator coupling to the preBötzinger complex. Nat Neurosci 12:1028-1035. CrossRef Medline

Todd AJ, Watt C, Spike RC, Sieghart W (1996) Colocalization of GABA, glycine, and their receptors at synapses in the rat spinal cord. J Neurosci 16:974-982. Medline

van Brederode JF, Yanagawa Y, Berger AJ (2011) GAD67-GFP+ neurons in the Nucleus of Roller: a possible source of inhibitory input to hypoglossal motoneurons. I. Morphology and firing properties. J Neurophysiol 105: 235-248. CrossRef Medline

Wang H, Stornetta RL, Rosin DL, Guyenet PG (2001) Neurokinin-1 receptor-immunoreactive neurons of the ventral respiratory group in the rat. J Comp Neurol 434:128-146. CrossRef Medline

Winter SM, Fresemann J, Schnell C, Oku Y, Hirrlinger J, Hülsmann S (2009) Glycinergic interneurons are functionally integrated into the inspiratory network of mouse medullary slices. Pflugers Archiv 458:459-469. CrossRef Medline

Yuste R, Denk W (1995) Dendritic spines as basic functional units of neuronal integration. Nature 375:682-684. CrossRef Medline 